\title{
Carving and Replaying Differential Unit Test Cases from System Test Cases
}

\author{
Sebastian Elbaum, Member, IEEE, Hui Nee Chin, \\ Matthew B. Dwyer, Member, IEEE, and Matthew Jorde
}

\begin{abstract}
Unit test cases are focused and efficient. System tests are effective at exercising complex usage patterns. Differential unit tests (DUTs) are a hybrid of unit and system tests that exploits their strengths. They are generated by carving the system components, while executing a system test case, that influence the behavior of the target unit and then reassembling those components so that the unit can be exercised as it was by the system test. In this paper, we show that DUTs retain some of the advantages of unit tests, can be automatically generated, and have the potential for revealing faults related to intricate system executions. We present a framework for carving and replaying DUTs that accounts for a wide variety of strategies and trade-offs, we implement an automated instance of the framework with several techniques to mitigate test cost and enhance flexibility and robustness, and we empirically assess the efficacy of carving and replaying DUTs on three software artifacts.
\end{abstract}

Index Terms-Automated test generation, carving and replay, regression testing.

\section{INTRODUCTION}

Software engineers develop unit test cases to validate Sindividual program units (e.g., methods, classes, and packages) before they are integrated into the whole system. By focusing on an isolated unit, unit tests are not constrained or influenced by other parts of the system in exercising the target unit. This smaller scope for testing usually results in more efficient test execution and fault isolation relative to full system testing and debugging [1], [20]. Unit test cases are also key components of several development and validation methodologies, such as extreme programming (XP) [2], test-driven development (TDD) practices [3], continuous testing [36], and efficient test prioritization and selection techniques [32].

Developing effective suites of unit test cases presents a number of challenges. Specifications of unit behavior are usually informal and are often incomplete or ambiguous, leading to the development of overly general or incorrect unit tests. Furthermore, such specifications may evolve independently of implementations requiring additional maintenance of unit tests even if implementations remain unchanged. Testers may find it difficult to imagine sets of unit input values that exercise the full range of unit behavior and thereby fail to exercise the different ways in which the unit will be used as a part of a system. An alternative approach to unit test development, which does not rely on specifications, is based on the analysis of a unit's implementation. Testers developing unit tests in this way may focus, for example, on achieving coverage-adequacy

- The authors are with the Department of Computer Science and Engineering, University of Nebraska, Lincoln, NE 68588.

E-mail: \{elbaum,dwyer\}@cse.unl.edu, \{huinee, majorde\}@gmail.com.

Manuscript received 28 Dec. 2007; revised 18 Aug. 2008; accepted 28 Aug. 2008; published online 2 Dec. 2008.

Recommended for acceptance by P. Frankl.

For information on obtaining reprints of this article, please send e-mail to: tse@computer.org, and reference IEEECS Log Number TSE-2007-12-0356.

Digital Object Identifier no. 10.1109.TSE.2008.103. criteria in testing the target unit's code. Such tests, however, are inherently susceptible to errors of omission with respect to specified unit behavior and may thereby miss certain faults. Finally, unit testing requires the development of test harnesses or the setup of a testing framework (e.g., JUnit [19]) to make the units executable in isolation.

Software engineers also develop system tests, usually based on documents that are available for most software systems that describe the system's functionality from the user's perspective, for example, requirement documents and user's manuals. This makes system tests appropriate for determining the readiness of a system for release or its acceptability to customers. Additional benefits accrue from testing system-level behaviors directly. First, system tests can be developed without an intimate knowledge of the system internals, which reduces the level of expertise required by test developers and makes tests less sensitive to implementation-level changes that are behavior preserving. Second, system tests may expose faults that unit tests do not, for example, faults that emerge only when multiple units are integrated and jointly utilized. Finally, since they involve executing the entire system, no individual harnesses need to be constructed.

While system tests are an essential component of all practical software validation methods, they do have several disadvantages. They can be expensive to execute; for large systems, days or weeks, and considerable human effort may be needed for running a thorough suite of system tests [25]. In addition, even very thorough system testing may fail to exercise the full range of behavior implemented by a system's particular units; thus, system testing cannot be viewed as an effective replacement for unit testing. Finally, fault isolation and repair during system testing can be significantly more expensive than during unit testing.

The preceding characterization of unit and system tests, although not comprehensive, illustrates that system and unit tests have complementary strengths and that they offer 
a rich set of trade-offs. In this paper, we present a general framework for the carving and replaying of what we call differential unit tests (DUTs) which aim to exploit those trade-offs. We termed them differential because their primary function is to detect differences between multiple versions of a unit's implementation. DUTs are meant to be focused and efficient like traditional unit tests, yet they are automatically generated along with a custom test-harness making them inexpensive to develop and easy to evolve. In addition, since they indirectly capture the notion of correctness encoded in the system tests from which they are carved, they have the potential for revealing faults related to complex patterns of unit usage.

In our approach, DUTs are created from system tests by capturing components of the exercised system that may influence the behavior of the targeted unit and that reflect the results of executing the unit; we term this carving because it involves extracting the relevant parts of the program state corresponding to the components exercised by a system test. Those components are automatically assembled into a test harness that establishes the prestate of the unit that was encountered during system test execution. From that state, the unit is replayed and the resulting state is queried to determine if there are differences with the recorded unit poststate.

Ideally, a set of DUT will

1. retain the fault detection effectiveness of system tests on the target unit,

2. execute faster or use fewer resources than system tests, and

3. be applicable across multiple system versions.

In addition, for program changes that are behavior preserving, effective DUTs will

4. report few differences that are not indicative of actual differences in system test results.

For changes that are intentionally behavior modifying, DUTs will, of course, detect differences. Rather than simply indicating that a difference is detected, our approach is able to provide a fine-grained view of the differences through the unit test outcomes. Using this information, developers will be able to quickly spot the effect of their intended modifications and to see where errors have been introduced.

In this paper, we investigate DUT carving and replay (CR) techniques with respect to the four numbered criteria. Through a set of controlled empirical studies within the context of regression testing, we compare the cost and effectiveness of system tests and carved unit tests. The results indicate that carved test cases can be as effective as system test cases in terms of fault detection, but much more efficient in the presence of localized changes. When compared against emerging work on providing automated extraction of powerful unit tests from system executions, [26], [29], [34], [41], the contributions of this paper are

- a framework for automatically carving and replaying DUTs that accounts for a wide variety of implementation strategies with different trade-offs,
- a novel state-based automated instantiation of the framework for CR at a method level that offers a range of costs, flexibility, and scalability, and

- an empirical assessment of the efficiency and effectiveness of CR of DUTs on multiple versions of three Java artifacts.

We note that this paper is a revised version of an earlier paper presented at the Foundations of Software Engineering Symposium 2006 [17] that includes various framework extensions presented in the next section, a more complete and detailed implementation presented in Section 3, and additional assessments described in Section 4. The related work is summarized in Section 5, and we outline several directions for future work in Section 6.

\section{A Framework for Test Carving and Replay}

Java programs can have millions of allocated heap instances [15] and hundreds of thousands of live instances at any time. Consequently, carving the raw state of real programs is impractical. We believe that cost-effective CR-based testing will require the application of multiple strategies that select information in raw program states and use that information to trade a measure of effectiveness to achieve practical cost. Strategies might include, for example, carving a single representative of each equivalence class of program states or pruning information from a carved state on which a method under test is guaranteed not to depend. The space of possible strategies is vast and a general framework for CR testing will aid in exploring possible cost-effectiveness tradeoffs in the space of CR testing techniques.

For the purposes of explaining our framework, we consider a Java program to be a state transition system. At any point during the execution of a program, the program state $\mathcal{S}$ can be defined conceptually as all of the values in memory. A program execution can be formalized either as a sequence of program states or as a sequence of program actions that cause state changes. A sequence of program states is written as $\sigma=s_{0}, s_{1}, \ldots$, where $s_{i} \in \mathcal{S}$ and $s_{0}$ is the initial program state as defined by Java. A state $s_{i+1}$ is reached from $s_{i}$ by executing a single action (e.g., bytecode). A sequence of program actions is written as $\bar{\sigma}$. We denote the final state of an action sequence $s(\bar{\sigma})$.

Regardless of how one develops, or generates, a unit test, there are four essential steps:

1. identify a program state from which to initiate testing,

2. establish that program state,

3. execute the unit from that state, and

4. judge the correctness of the resulting state.

In the rest of this section, we define a general framework that allows different strategies to be applied in each of these steps.

\subsection{Basic Carving and Replaying}

Fig. 1 illustrates the general CR process. Given a system test case $s t_{x}$, carving a unit test case $D U T_{x m}$ for target unit $m$ during the execution of $s t_{x}$ consists of capturing $s_{\text {pre }}$, the program state immediately before the first instruction of an activation of method $m$, and $s_{\text {post }}$, the program state immediately after the final instruction of $m$ has executed. 
- Given system test case $s t_{x}\{$ input/s, expected output/s $\}$

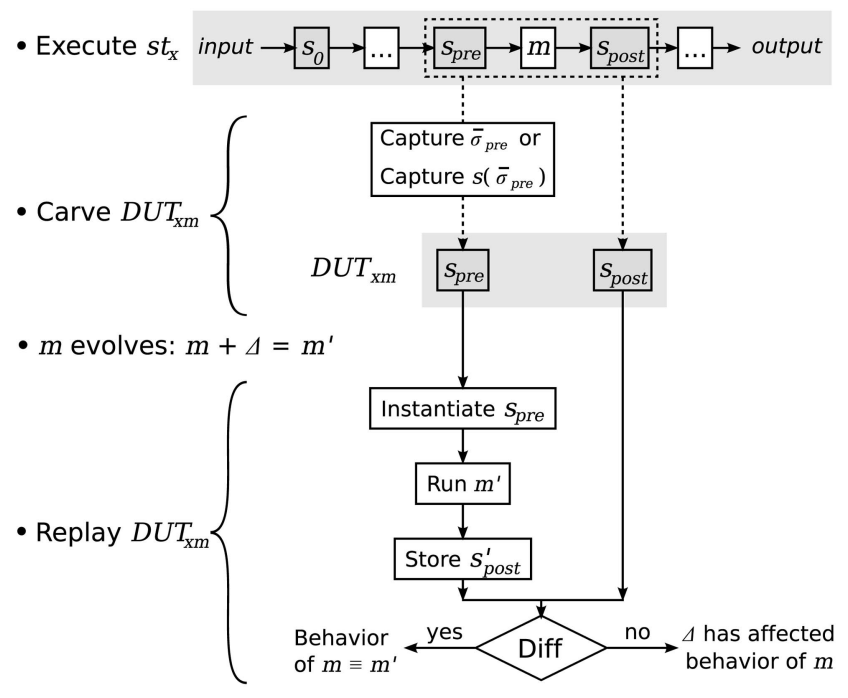

Fig. 1. Carving and replay process.

The captured pair of states $\left(s_{\text {pre }}, s_{\text {post }}\right)$ defines the DUT case for method $m$, denoted $D U T_{x m}$. States in this pair can be defined by directly capturing a pair of states in $\sigma$ or by recording the cumulative effects of sequences of program actions $\bar{\sigma}_{\text {pre }}$ and $\bar{\sigma}_{\text {post }}$, i.e., recording $s\left(\bar{\sigma}_{\text {pre }}\right)$ and $s\left(\bar{\sigma}_{\text {post }}\right)$. A $\mathrm{CR}$ testing approach is said to be state based if it records pairs $\left(s_{\text {pre }}, s_{\text {post }}\right)$ and action based if it records pairs $\left(\bar{\sigma}_{\text {pre }}, \bar{\sigma}_{\text {post }}\right)$. We note that action-state hybrid CR approaches that record, for example, pairs of actions sequences and states $\left(\bar{\sigma}_{\text {pre }}, s_{\text {post }}\right)$ may also be useful.

In practice, it is common for a method $m$ to undergo some modification (e.g., to $\mathrm{m}^{\prime}$ ) over the program lifetime. To efficiently validate the effects of a modification, we replay $D U T_{x m}$ on $m^{\prime}$. Replaying a DUT for a method $m^{\prime}$ requires the instantiation of $s_{\text {pre }}$ by either loading the state $s_{\text {pre }}$ into memory or by executing $\bar{\sigma}_{\text {pre }}$, depending on how the state was carved. From this state, execution of $m^{\prime}$ is initiated and it continues until it reaches the point corresponding to the carved $s_{\text {post }}$. At that point, the current execution state $s_{\text {post }}^{\prime}$ is compared to $s_{\text {post }}$. If the post states are the same, we can attest that the change did not affect the behavior of the target unit exercised by $D U T_{x m}$. However, if the change altered the semantics of $m$, then further processing will be required to determine whether the alteration matches the developer's expectations (we discuss the support that provided by our implementation of CR in Section 2.4).

This basic CR approach suffers from several fundamental limitations that must be addressed in order to make CR cost-effective. First, the proposed basic carving procedure is at best inefficient and likely impractical. It is inefficient because a method may only depend on a small portion of the program state, thus storing the complete state is wasted effort. Furthermore, two distinct complete program states may be identical from the point of view of a given method, thus carving complete states would yield redundant unit tests. It is impractical because storing the complete state of a program may be prohibitively expensive in terms of time and space. Second, changes to $m$ may render $D U T_{x m}$ unexecutable in $m^{\prime}$. Reducing the cost of CR testing is important, but we must produce DUTs that are robust to various types of changes so that they can be executed across a series of system versions in order to recover the overhead of carving, and provide further support to analyze the reasons behind DUTs detected differences. Finally, the use of complete poststates to detect behavioral differences is not only inefficient but may also be too sensitive to behavior differences caused by reasons other than faults (e.g., fault fixes, algorithm improvements, and internal refactoring) leading to the generation of brittle tests. The following sections address these challenges.

\subsection{Improving CR with Projections}

We focus CR testing on a single method by defining projections on carved prestates that preserve information related to the unit under test and are likely to provide significant reduction in prestate size.

State-based projections. A state projection function $\pi$ : $\mathcal{S} \rightarrow \mathcal{S}$ preserves specific program state components and elides the rest. For example, a state projection may preserve the scalar fields in the object, a subset of the references to other objects, or a combination of both. Underlying many useful state projections is the notion of heap reachability. An object $o^{\prime}$ is reachable in one dereference from object $o$ if the value of some field $f$ references $o^{\prime}$; let reach $(o)=\left\{o^{\prime} \mid \exists_{f \in \text { Fields }(\operatorname{Class}(o))}\right.$ o. $\left.f=\operatorname{address}\left(o^{\prime}\right)\right\}, \quad$ w h e r e Fields $(c)$ denotes the set of (nonstatic) fields defined for class $c$ and Class returns the class of an object.

Objects reachable through any chain of dereferences up to length $k$ from $o$ are defined by using the iterated composition of this binary relation, $\bigcup_{1 \leq i \leq k}$ reach $^{i}(o)$; as a notational convenience, we will refer to this as $\operatorname{reach}^{k}(o)$. The positive transitive closure of the relation, $\operatorname{reach}^{+}(o)$, defines the set of all reachable objects from $o$ in one or more dereferences.

To promote replay capabilities, state-based CR testing approaches at the method level should use projections that retain at most the set of heap objects reachable from a given calling context. That set includes heap objects reachable through the receiver object, the call's parameters, static fields within the method's class, and public static fields from other classes. More formally, given a call $\operatorname{r.m}\left(p_{1}, \ldots, p_{n}\right)$, the reachable objects from the calling context include

1. reach $^{+}(r)$,

2. $\left\{o \mid \exists_{i \in 1 \ldots n} o \in \operatorname{reach}^{+}\left(p_{i}\right)\right\}$,

3. $\left\{o \mid \exists_{f \in \text { Fields }_{s}\left(\text { Class }_{(r))} o \in \text { reach }^{+}(f)\right\} \text { where Fields }}\right.$ is the set of static fields for a class and reach has been extended to fields, and

4. $\left\{o \mid \exists_{\text {cflass }(m)} \exists_{f \in \text { Fields }_{p s}(c)} o \in \operatorname{reach}^{+}(f)\right\} \quad$ w h e r e Fields $s_{p s}$ is the set of public static fields for a class and Class is the class declaring a given method.

This projection is lossless for reasoning about a method invocation since it retains all of the information in $s_{\text {pre }}$ that could possibly be accessed by the call to $m$.

More efficient projections might consider a subset of the heap elements captured by the calling context reachable projection. Some of these projections will use a notion of distance to determine what heap elements to preserve (e.g., retain all the heap elements that may be reached in up to 


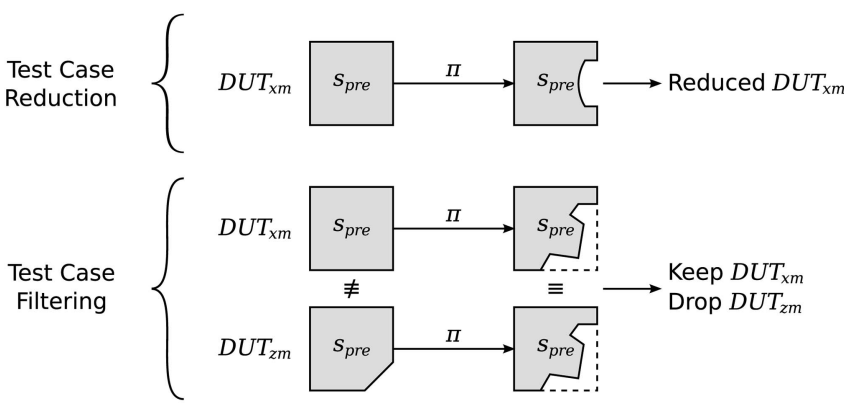

Fig. 2. Application of projections.

$k$ dereferences) while others may aim to maintain just the basic heap content and the heap structure (e.g., retain only the values of reference fields, thereby eliminating all scalar fields, which would maintain the heap shape of a program state). Some projections will determine the portion of the state to preserve ahead of time through some form of source code analysis (e.g., side-effects analysis or reachability analysis), while others will make that determination at runtime (e.g., retain the heap elements reachable or read during execution).

The range of projections makes it possible to trade robustness for reduction in carving cost and replaying time by defining projections that eliminate more state information. Section 3.2 presents five projections that exercise this trade-off.

Action-based projections and transformations. Projections can also operate on sequences of program actions, $\bar{\pi}: \bar{\sigma} \rightarrow \bar{\sigma}$, to distill the portion of a program run that affects the prestate of a unit method. Unfortunately, a purely action-based approach to state capture will not work for all Java programs. For example, a program that calls native methods does not, in general, have access to native method instructions. To accommodate this, we can allow for transformation of actions during carving, i.e., replace one sequence of instructions with another. Transformation could be used, for example, to replace a call to a native method with an instruction sequence that implements the side effects of the native method. More generally, one could design an instance of $\bar{\pi}$ that would replace any trace portion with a summarizing action sequence.

Applying projections. Fig. 2 illustrates two potential applications of projections on DUTs: test case reduction and test cases filtering.

Reduction aims at thinning a single carved test case by retaining only the projected prestate (in Fig. 2, for example, the projection on $s_{\text {pre }}$ carved from $D U T_{x m}$ leads to a smaller $s_{\text {pre }}$ ). Reducing a DUT's prestate results in smaller space requirements and, more importantly, in quicker replay since loading time is a function of the prestate size. For example, a method like totalPages in Fig. 3 that returns the int field pages presents a clear opportunity to benefit from a reduction that retains just the scalar fields. Such reduction would avoid the need to load some potentially large objects such as the info hashtable, making replay faster. Depending on the type of projection, such gains may be achieved at the expense of additional analysis and carving time (e.g., using a more precise but expensive analysis to determine what to

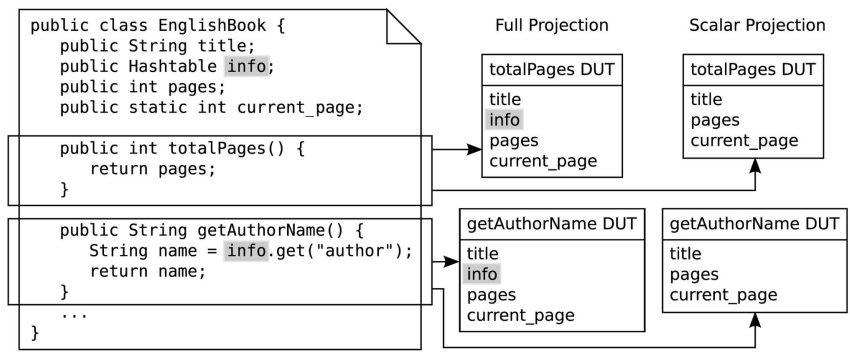

Fig. 3. Reduction and test executability.

carve), or reduced fault detection power (e.g., a projection may discard an object that was necessary to expose the fault). Furthermore, test executability may be sacrificed as well when, for example, the data structures needed to successfully instantiate the object in memory become unavailable due to applied projections. In Fig. 3, the relevant program state for getAuthorName includes the field info of type java.util. Hashtable from the EnglishBook class, which stores the type of information for a particular book (such as publication date and author name). If the same scalar-only type reduction were applied, then DUTs for getAuthorName would not be replayable because the info field would be missing from the prestate. Under this circumstance, an alternative projection to enable reduction could aim for carving the fields of the parts of the hashtable just touched during the execution. The key is to identify the suitable level of reduction that would maximize efficiency, fault detection, and test executability at the same time.

Filtering aims at removing redundant DUTs from the suite. Consider a method that is invoked during program initialization and is independent of the program parameters. Such a method would be exercised by all system tests in the same way and likely result in multiple identical DUTs for that particular method. Filtering by comparing complete prestates could remove such duplicate tests, retaining just the DUTs that have a unique $s_{\text {pre }}$. Consider a simple accessor method with no parameters that returns the value of a scalar field. If this method is invoked by tests from different prestates, then multiple DUTs will be carved, and filtering based on complete prestates will retain all of the DUTs even though they exercise the same behavior. For this method, filtering based on a projection that preserves just the subset of a prestate that is reachable from this in one dereference may remove multiple redundant DUTs (in Fig. 2, $\pi\left(s_{p r e}\right)$ for $D U T_{x m}$ and for $D U T_{z m}$ are identical so one of them can be removed). Clearly, in some cases, overaggressive filtering may result in a lower fault detection capability since we may discard a DUT that is different and, hence, potentially valuable.

Note that, contrary to test case reduction, while filtering may sometimes only consider subsets of program states to judge equivalence, the stored program states are not modified; consequently, test executability is preserved since the DUTs that are retained are complete. In practice, however, reduction and filtering are likely to be applied in tandem such that reduced tests are then filtered or filtered tests are then reduced (without necessarily using the same projection for reduction and filtering). 


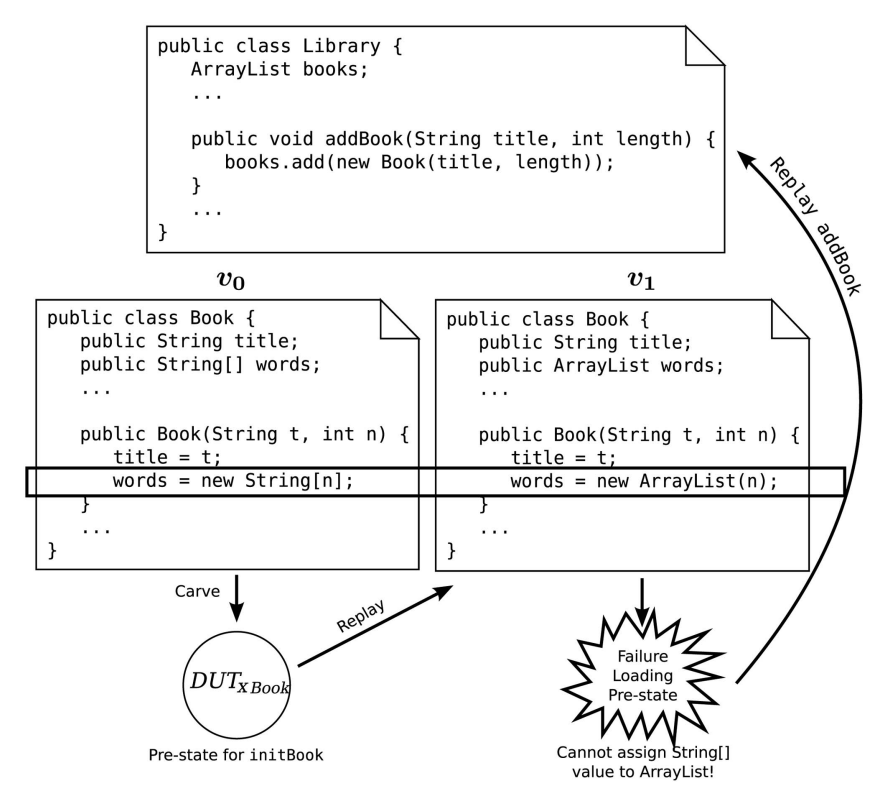

Fig. 4. An example of replay failure.

\subsection{Strategies to Manage Replay Anomalies}

We have discussed how overly aggressive reductions can impair replay. Similarly, certain method changes such as modifications in a method's signature or key data structures may prevent a DUT from correct replay. For example, consider the scenario shown in Fig. 4 where we carved $D U T_{x B o o k}$ from $v_{0}$ of the Book class. Replaying the constructor for Book with the carved $D U T_{x B o o k}$ in $v_{1}$ encounters an error resulting from incompatible types in the words field between versions. Effective CR testing must detect failures arising from carving limitations, differentiate them from regular application failures, and find ways to use this information to further guide the testing process and ensure the coverage of the target method. The detection and differentiation steps are implementation specific and are discussed in the following section, while this section focuses on what to do once a DUT fails to replay.

When $D U T_{x m}$ cannot be replayed, one could replay the system test case $s t_{x}$ on the new version of the software, while carving a new $D U T_{x m}$ to replace the one invalidated by the program modification. The idea here is to use the DUT failure as a trigger for the system test case execution to ensure the proper coverage of the target method by the system test while creating DUTs for the future. An alternative approach that avoids system test case execution and immediate recarving takes advantage of the existing body of executable DUTs on other methods that exercise the target method. For instance, in Fig. 4, replaying the DUTs for the addBook method of the Library class would exercise the Book constructor (through the invocation of new Book(title, length)) without the explicit loading of $D U T_{x B o o k}$. This approach is appealing because it eliminates the immediate need for recarving while still enabling the localized execution of a changed DUT. However, it does not account for the potential existence of multiple callers and the possibility that some callers may not be replayable themselves.
When $D U T_{x m}$ fails, we can identify a set of DUTs whose execution reaches $D U T_{x m}$ 's prestate; we call such a set a replay frontier of $D U T_{x m}$. There may be many replay frontiers for a given DUT. Selection of an appropriate frontier is guided by three criteria: 1) the ability of the frontier to successfully replay the behavior exercised in $\left.D U T_{x m}, 2\right)$ the cost of executing the frontier, and 3) the localization of defect detection relative to $D U T_{x m}$.

At one extreme, $D U T_{\text {xmain, }}$ i.e., the main program, comprises a replay frontier for any DUT. Intuitively, it maximizes replayability, since it is essentially an execution of system test case $s t_{x}$. On the other hand, this frontier will be more costly to execute than other frontiers and will provide a less focused characterization of detected defects. At the other extreme, one could identify the set of DUTs that directly invoke method $m$ corresponding to the failed DUT. Executing these DUTs will provide localized replay of the behavior of $D U T_{x m}$ and may be significantly less expensive than $D U T_{x m a i n}$. This frontier is more likely to exhibit replay anomalies due to the proximity to the change (e.g., when the caller and callee are methods in the same changed class).

In Section 3.1, we explore a family of strategies that attempt to balance the three frontier selection criteria.

\subsection{Adjusting Sensitivity through Differencing Functions}

The basic CR testing approach described earlier compares a carved complete poststate to a poststate produced during replay to detect behavioral differences in a unit. The use of complete poststates is both inefficient and unnecessary for the same reasons as outlined above for prestates. While we could use comparison of poststate projections to address these issues, we believe that there is a more flexible solution that could also help control DUTs' sensitivity to changes.

Method unit tests are typically structured so that, after a sequence of method calls that establish a desired prestate, the method under test is executed. When it returns, additional method calls and comparisons are executed to implement a pseudo-oracle. For example, unit tests for a redblack tree might execute a series of insert and delete calls and then query the tree height and compare it to an expected result to judge partial correctness. We allow a similar kind of pseudo-oracle in CR testing by defining differencing functions on poststates that preserve selected information about the results of executing the unit under test. These differencing functions can take the form of poststate projections or can capture properties of poststates, such as tree height or size, and consequently may greatly reduce the size of poststates while preserving information that is important for detecting just the meaningful behavioral differences.

We define differencing functions that map states to a selected differencing domain, dif $: \mathcal{S} \rightarrow \mathcal{D}$. Differencing in CR testing is achieved by evaluating $\operatorname{dif}\left(s_{\text {post }}\right)=\operatorname{dif}\left(s_{\text {post }}\right)$. State projection functions are simply differencing functions where $\mathcal{D}=\quad \mathcal{S}$. In addition to the reachability projections defined in the previous section, projections on unit method return values, called return differencing, and on fields of the unit instance referenced by this, called instance differencing, are useful since they correspond to techniques used widely in hand-built unit tests. 


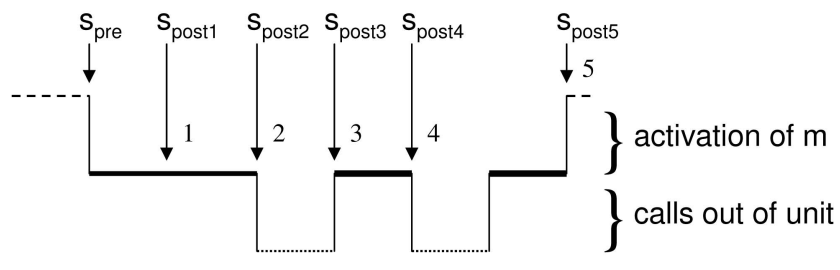

Fig. 5. Differencing sequences of poststates.

A central issue in differential testing is the degree to which differencing functions are able to detect changes that correspond to faults while masking implementation changes. We refer to this as the sensitivity of a differencing function. Clearly, comparing complete poststates will be highly sensitive, detecting both faults and implementation changes. A projection function that only records the return value of the method under test will be insensitive to implementation changes while preserving some fault sensitivity. Note also that these differencing functions provide different incomplete views of the program state. Their incompleteness reduces cost and may add some level of insensitivity to changes in the implementation, but it could also reduce their fault detection effectiveness. We address this by allowing for multiple differencing functions to be applied in CR testing which has the potential to increase fault sensitivity, without necessarily increasing implementation change sensitivity. For example, using a pair of return and instance differencing functions allows one to detect faults in both instance field updates and method results, but will not expose differences related to deeper structural changes in the heap. Fault isolation efficiency could also be enhanced by the availability of multiple differencing functions since each could focus on a specific property or set of program state components that will help developers focus their attention on a potentially small portion of program state that may reflect the fault.

DUTs can also be refined to increase their sensitivity in the temporal dimension by capturing sequences of poststates $\left(s_{\text {pre }}, \sigma_{\text {post }}\right)$ that capture intermediate points during the execution of the method under test. Such poststate sequences can be valuable to support fault isolation and debugging efforts since they provide additional observability on program states generated during the method execution. Fig. 5 illustrates a scenario in which a DUT begins execution of $m$ at $s_{\text {pre }}$. Conceptually, during replay, a sequence of poststates is differenced with corresponding states at intermediate states of the method under test. For example, at point 1 , the test compares the current state to the captured $s_{\text {post } 1}$, similarly at points 2 and 3 the pre and poststates of the call out of the unit are compared.

Using a sequence of poststates requires that a correspondence be defined between locations in $m$ and $m^{\prime}$. Correspondences could be defined using a variety of approaches, for example, one could use the calls out of $m$ and $m^{\prime}$ to define points for poststate comparison (as is illustrated in Fig. 5) or common points in the text of $m$ and $m^{\prime}$ could be detected via textual differencing. Fault isolation information is enriched by using multiple poststates, since if the first detected difference is at location $i$, then that difference was introduced in the region of execution between location $i-1$ and $i$. Of course, storing multiple poststates may be expensive so its use can only be advocated to narrow the scope of code that must be considered for fault isolation once a behavioral difference is attributed to a fault.

\section{INSTANTIATING THE FramewORK}

In this section, we describe the architecture and implementation details of a state-based instantiation of the framework for the Java programming language. Section 5 discusses alternative CR implementations.

\subsection{System Architecture}

Fig. 6 illustrates the architecture of the CR infrastructure. The carving activity starts with the Carver class which takes four inputs: the program name, the target method(s) $m$ within the program, the system test case $s t_{x}$ inputs, and the reduction and filtering options.
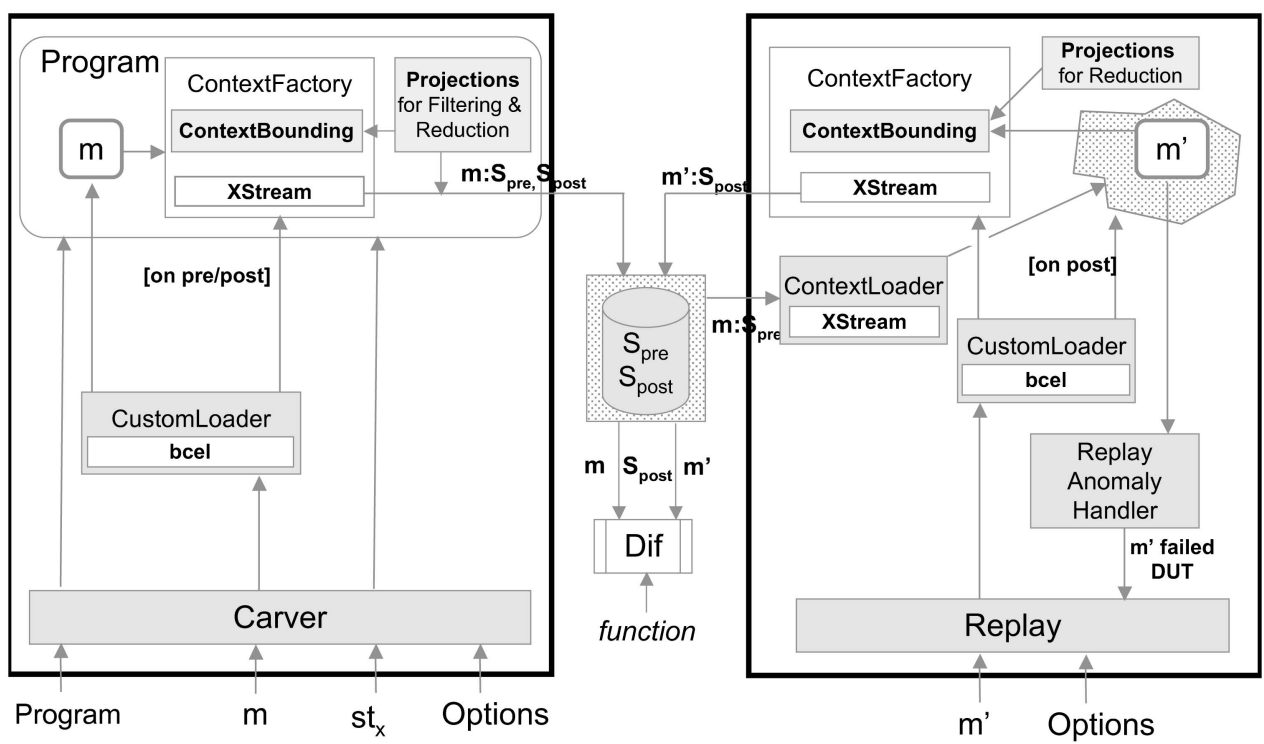

Fig. 6. CR tool architecture. 
Carver utilizes a custom class loader CustomLoader (that employs the Byte Code Engineering Library (BCEL) [14]) to incorporate instrumentation code into the program. We instrument the bytecode for all loaded program classes except the ones that are in the Java API, part of the CR tool, and members of third-party libraries used by the tool. The instrumentation uses the singleton ContextFactory class to store pre and poststates of program methods at the entry and exit points of the methods (including exceptional exit points forced by throw instructions in $m$ or methods called by $m$ ). Every execution of a method that is targeted for CR testing will lead to, at least, two invocations of the ContextFactory: one at the entry point of the method to store $s_{\text {pre }}$ and one at the exit point of the method to store $s_{\text {post }}$.

As discussed earlier, carving the entire state of the program is impractical, therefore the ContextFactory utilizes ContextBounding to determine the parts of the program state to be stored during carving based on the chosen projections to perform reduction and filtering. Once the carving scope has been determined, ContextFactory utilizes an open source package, XStream [40], to perform the serialization to XML of the heap objects in the defined scope. Finally, ContextFactory stores the serialized program states. By default, ContextBounding applies the most conservative projection: an interface reachability projection (as described in Section 2.2), and filters DUTs based on that projection. Several other projections and lossy filters are available and introduced in the upcoming sections.

While XStream is a powerful object serialization package, by default it does not serialize a class's static fields. However, to truly replay a method with the prestate that it encountered during a system test, we need to establish the values of static fields as well as instance fields since both influence the execution of the method. Fortunately, XStream allows a high level of customization. We implemented a custom extension for XStream that enables the serialization of and the application of projections to static fields by retrieving their contents including transitively reachable objects, serializing it using XStream, and placing the resulting XML in a special tag which we introduced to contain static fields. This XStream extension also takes care of deserializing the static fields and restoring them upon full object deserialization.

We have implemented two options for storing poststates: 1) complete poststate descriptions encoded in an XML format and 2) unique fingerprints of poststates defined by hashing of XML encodings. The complete representation is helpful in determining which part of the poststate was affected by the program changes, but carving execution time and storage requirements may be higher. Fingerprint storage allows for more efficient carving, storage, and difference detection, but does not allow for a detailed characterization of state differences.

The other primary CR component, Replay, shares many of the core classes with Carver (CustomLoader, ContextBounding, ContextFactory) and works in a similar manner. To establish the desired prestate on which to invoke $m^{\prime}$, Replay utilizes the ContextLoader class to obtain and load the carved $s_{\text {pre }}$ of $m$, using XStream to deserialize the stored state. After that, $m^{\prime}$ is invoked. Similar to Carver, Replay instruments the class of $m^{\prime}$ and utilizes the ContextFactory, but only to store $s_{\text {post }}$

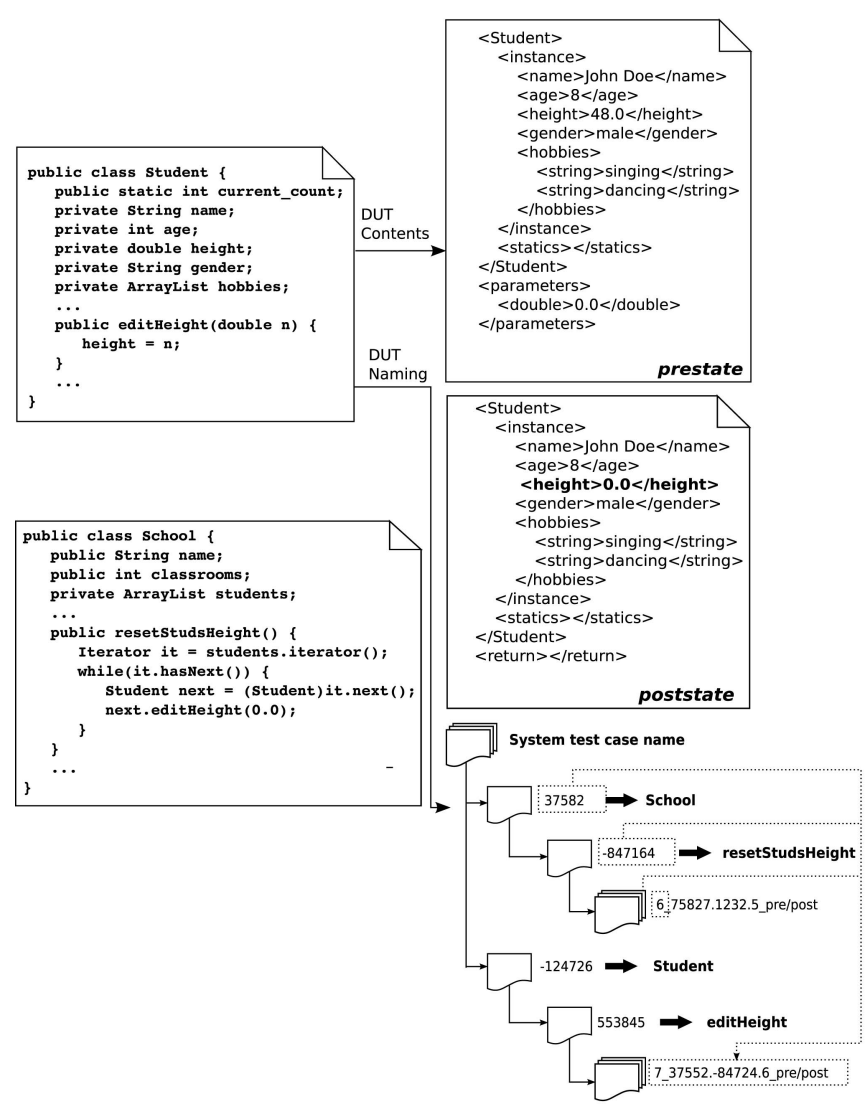

Fig. 7. DUT file contents and directory structure.

after $m^{\prime}$ is invoked. Once $m^{\prime}$ has been replayed, we use Dif, the differencing mechanism, to compare the $s_{\text {post }}$ of $\mathrm{m}^{\prime}$ generated during Replay with the carved $s_{\text {post }}$ of $m$ to determine whether the changes in $m^{\prime}$ resulted in a behavioral difference. Currently, we have fully automated the differencing functions on return values, instance fields, state fingerprints, and complete XML state encodings which include static fields.

If Replay fails for $m^{\prime}$, the ReplayAnomalyHandler will begin the process of exploring the replayable frontier of $m^{\prime}$. The current implementation to explore the frontier can use call graphs or the DUTs built-in caller information to guide the replay process in the presence of an anomaly. These two mechanisms trade carving efficiency for replay efficiency. Keeping track of the DUT caller information requires an additional tracking method within ContextFactory that maintains a DUT call stack which increases carving overhead and storage per DUT. However, such information often leads to a more precise determination of what DUT needs to be replayed in the presence of a replay failure, which can cut down replay time.

Each generated DUT is composed of two files: prestate, which includes the objects reachable through the method's parameters or the class fields, and poststate, which contains the reachable objects and return value for the method. DUTs are organized through a directory structure of four levels that includes a level for system tests, a level for classes, a level for methods, and one for DUTs. The DUTs are assigned an identification hashcode based on their corresponding method signature as well as the information identifying its caller DUT. Fig. 7 provides an example of DUT file contents for the 
method editHeight of the class Student and illustrates the DUT naming scheme described above.

\subsection{Implemented Projections}

Here, we describe the types of projections implemented in the CR tool. These offer a degree of control over the carved test cases that can be generated.

Interface k-bounded reachable projection. The interface $k$-bounded reachable projection for a method invocation $r . m\left(p_{1}, \ldots, p_{n}\right)$ defines the set of preserved objects to include only those reachable from the target method class via dereference chains of length up to $k$, i.e., $\operatorname{reach}^{k}(r) \cup$

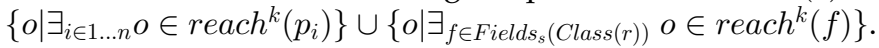
The intuition behind this projection is that DUTs that have identical heap structure up to depth $k$ may exercise $m$ in a similar manner and this could lead to significant filtering (e.g., a method working on link lists may only need to access the first $k$ elements in a list to exhibit all of its interesting behavior). Using small values of $k$ can greatly reduce the size of the recorded prestate and, in turn, this can lead to more DUTs being judged equivalent. For many methods, a small value of $k$ will have no impact on unit-test robustness. For example, a value of 1 would suffice for a method whose only dereferences are accesses to fields of this.

If a method changes to access data along a reference chain of length greater than the $k$ set during carving, then the DUTs carved using the $k$-bounded projection would have retained insufficient data about the prestate to allow replay. Our implementation dynamically detects this situation and raises a custom exception to indicate a replay anomaly. During state storage, the heap is traversed and objects that are referenced at a depth of $k+1$, but no shallower, are marked. For each such marked objects, a sentinel attribute is introduced into the prestate XML encoding. When the prestate is deserialized, every object created from XML with a sentinel attribute is added to a Collection. Instrumentation is added after all GETFIELD instructions to check for the membership of the requested object in the Collection. If the object is a member, the instrumentation throws a SentinelAccess Exception. This prevents NullPointerExceptions from being thrown during sentinel object accesses which could be confused with normal application exceptions. It also prevents invalid replay results which would be caused by a program handling a null value and continuing execution when the value would not normally have been null. These SentinelAccessExceptions are one mechanism for identifying replay anomalies and triggering the ReplayAnomalyHandler.

May-reference reachable projection. The may-reference reachable projection uses a static analysis that calculates a characterization of the objects that may be referenced by a method activation either directly or through method calls. This characterization is expressed as a set of regular expressions of the form: $p f_{1} \ldots f_{n}\left(F^{+}\right)$? which captures an access path that is rooted at a parameter $p$ and consists of $n$ dereferences by the named fields $f_{i}$ (e.g., p.next. next.val). If the analysis calculates that the method may reference an object through a dereference chain of length greater than $n$, the optional final term is included to capture objects that are reachable from the end of the chain through dereference of fields in the set $F$. In general, $F$ is calculated on a per-type basis where $F(c)$ is the subset of fields of class $c$ that may be referenced by an execution of the method. Let $\operatorname{reach}_{F}(o)=\left\{o^{\prime} \mid \exists_{f \in F(\operatorname{Class}(o))} o . f=\operatorname{address}\left(o^{\prime}\right)\right\} \quad$ c a p t u r e reachability restricted to the subset of fields encoded in $F$; reach $_{f}$ denotes reachability for the singleton set $\{f\}$. For a regular expression of the form $p f_{1} \ldots f_{m}$, where $m \leq n$, we construct the set: $\operatorname{reach}_{f_{1}}(p) \cup \ldots \cup \operatorname{reach}_{f_{m}}\left(\ldots\left(\operatorname{reach}_{f_{1}}(p)\right)\right)$, since we want to capture all references along the path. If the regular expression ends with the term $F^{+}$, then we union an additional term of the form reach $_{F}^{+}\left(\right.$reach $_{f_{m}}\left(\ldots\left(\right.\right.$ reach $\left.\left.\left._{f_{1}}(p)\right)\right)\right)$. This projection can reduce the size of carved prestates while retaining arbitrarily large heap structures that are relevant to the method under test.

We implement our projection using the context-sensitive interprocedural read-write analysis implemented in Indus [28]. This analysis handles all of the complexities of Java in its alias analyses including the safe approximation of readwrite operations performed in libraries. We configure this analysis to calculate $l$-bounded access path and then generate regular expressions that capture the set of all possible referenced access paths up to length $l$; we use a default of $l=2$. When traversing the program for serialization using XStream, we simultaneously keep track of all regular expressions and mark only those objects that lie on a defined access path for storage in XML. Note that the $l$ bounding controls the precision of the static analysis and does not limit the depth of the prestate carving, consequently no sentinel objects are introduced with this projection. This analysis is also capable of detecting when a method is side-effect free and in such cases the storage of poststates is skipped since method return values completely define the effect of such methods.

Touched-carving projection. The touched-carving projection utilizes dynamic information about all the fields that were read or written during the method execution (or the execution of methods called from that target method) to decide which parts of the program states to store. Our implementation of this projection starts with the instrumentation utilized by the interface $k$-bounded reachable projection, and it incorporates additional instrumentation to mark the parts of the heap referenced by the instrumented methods. During carving, the additional instrumentation helps to identify referenced fields and stores them. Fields that are not referenced are stored up to depth of $k$ to ensure a level of robustness in the event of method changes that result in references to additional fields.

There are two implementation aspects of this projection worth mentioning. First, given that we cannot know which fields will be read or written to prior to the execution of a method, we first store the method's complete $s_{\text {pre }}$ in memory, then execute an instrumented version of the method that records all referenced fields for storage in the DUT. This record is then used to write the XML structure or fingerprint to disk. Second, $D U T_{x m}$ 's $s_{\text {pre }}$ needs to store the fields referenced by $m$ and also the fields referenced by all the methods $m$ calls. To do this, we maintain object graphs during carving. Fig. 8 illustrates how this works for the class Person when a call to checkGrowth is made. The gray areas indicate fields that were referenced either directly or indirectly by the method. Fields in light gray were read, 


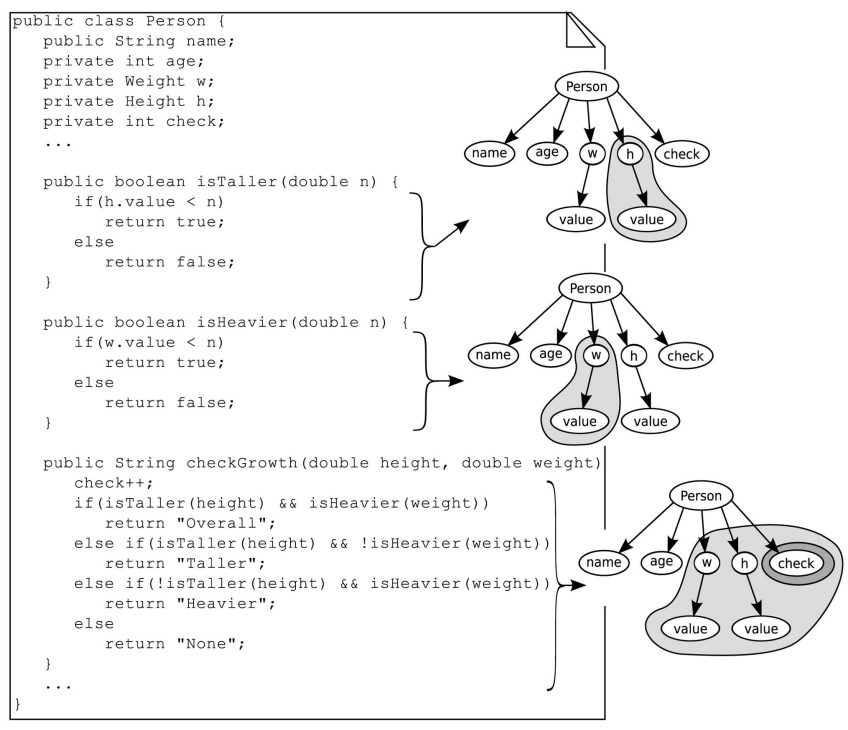

Fig. 8. Touched-carving projection. Light gray indicates read items and dark gray indicates written items.

fields in dark gray were written, and fields in both were read and written. In the method checkGrowth, the field check is both read and written in the first line. The fields $w, h$, w.value, and h.value are read indirectly through calls to isTaller and isHeavier.

Clustering projection. The clustering projection attempts to identify a set of similar DUTs, $D U T_{x \text { callee }, 1}, D U T_{x ! c a l l e e, 2}, \ldots$, that result from the repeated invocation of callee from within the same DUT, $D U T_{\text {xcaller, }}$ of method caller. Fig. 9 illustrates an instance where this projection may be very effective. Every invocation of printbook results in a $D U T_{\text {xprintbook }}$ and one DUT for incIndex for each iteration of the while loop, i.e., length $D U T s_{\text {xincIndex }}$. Consequently, there may be many DUTs generated for incIndex and the added value of those DUTs may be limited. Instead of carving such DUTs, through the clustering projection, we keep track of the number of invocations of incIndex from the context defined by $D U T_{\text {xprintBook }}$. When that number exceeds a predetermined threshold, we replace the incIndex DUTs with a reference to $D U T_{\text {xprintbook }}$ which enables their indirect replay. This projection amounts to a heuristic for identifying a replay frontier and exploiting that frontier to filter DUTs lower in the call hierarchy.

Normalizing transient data. Projections seek to retain relevant differences between states while eliminating data that is regarded as irrelevant. It is possible to eliminate differences, without eliminating data by normalizing values, for example, setting all java.util.Date fields to a fixed value, or fixing the seed in java.util.Random. In most Java programs, there are a wealth of data types that have transient data. We have identified a number of those types and applied normalizing value transformations. For example, autoflushing Flushable implementations can be flushed at different times and differences in the contents of the backing Buffer objects, char []s or byte []s can occur under normal circumstances. To normalize buffer array contents, we check for Flushable types and Buffer

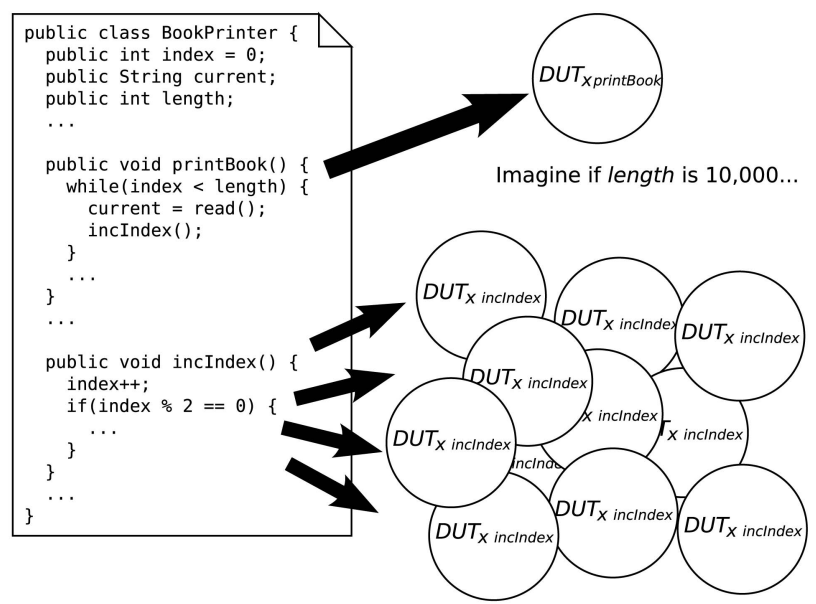

Fig. 9. A filtering strategy based on a caller's context.

types before serialization. If a Flushable type is found, the flush method is called, if a Buffer type is found, the clear method is called. Since the implementations for flush and clear do not truly clear the backing array (they just reinitialize a pointer), we use reflection to get all fields with type char [] or byte[] and overwrite them with zeros. Then, serialization continues as normal. This process guarantees that variable buffer contents are consistent across all poststates of multiple executions.

\subsection{Toolset Limitations}

The current CR toolset is robust in its support of the Java language and commonly used libraries and frameworks, but it has two limitations.

Threading limitations. Our toolset was originally developed for sequential programs and the instrumentation strategy we employ in the Carver is not thread safe. Rather than employ a basic locking strategy in instrumentation to assure thread-safety, we have deferred the treatment of thread-safety to pursue a more complex and potentially more efficient solution that avoids locking overhead in accessing Carver data structures. We note that, for replay, thread safety is not an issue.

Serialization limitations. Our approach requires the ability to save and restore object data representing the program state. However, the Java java.io.Serializable interface limits the type of objects that can be serialized. For example, Java designates file handler objects as transient (nonserializable) because it reasonably assumes that a handler's value is unlikely to be persistent and restoring it could enable illegal accesses. The same limitations apply to other objects, such as database connections and network streams. In addition, the Java serialization interface may impose additional constraints on serialization. For example, it may not serialize classes, methods, or fields declared as private or final in order to avoid potential security threats. Fortunately, we are not the first to face these challenges. We found multiple serialization libraries that offer more advanced and flexible serialization capabilities with various degrees of customization. We ended up choosing the XStream library [40] because it comes bundled with many converters for nonserializable types and a 
default converter that uses reflection to automatically capture all object fields, it serializes to XML which is more compact and easier to read than native Java serialization, and it has built-in mechanisms to traverse and manage the storage of the heap which was essential in implementing the projections. In cases where XStream support was insufficient, we developed custom extensions such as the one mentioned before that enables the serialization of static fields. We anticipate that further extensions and customizations will accommodate other special object types.

Scope limitations. Our toolset captures a large part of the program state relevant to a calling context, but it does not capture all of it. We do not capture public variables declared by other classes that are not reachable from the target method class. This implicit projection may cause false replay differences, but it is necessary to avoid bulky and inefficient DUTs. In addition, we do not capture fields declared static final since they cannot be restored during deserialization. However, we note that such fields are often initialized to fixed values that are consistent across executions, limiting their influence in post state differences.

\section{Empirical Study}

The goal of the study is to assess execution efficiency, fault detection effectiveness, and robustness of DUTs. We will perform such assessment through the comparison of system tests and their corresponding carved unit test cases in the context of regression testing. Within this context, we are interested in the following questions:

RQ1. Can DUTs reduce regression testing costs? We would like to compare the cost of carving and reusing DUTs versus the costs of utilizing regression test selection techniques that work on system test cases.

RQ2. What is the fault detection effectiveness of DUTs? This is important because saving testing costs while reducing fault detection is rarely an enticing tradeoff.

RQ3. How robust are the DUTs in the presence of software evolution? We would like to assess the reusability of DUTs on a real evolving system and examine how different types of change can affect the robustness and sensitivity of the carved tests.

\subsection{Regression Test Suites}

Let $P$ be a program, let $P^{\prime}$ be a modified version of $P$, and let $T$ be a test suite developed initially for $P$. Regression testing seeks to test $P^{\prime}$. To facilitate regression testing, test engineers may reuse $T$ to the extent possible. In this study, we considered five types of test regression techniques, two that directly reuse with system tests $(S)$ and three that reuse the DUTs carved from the system test suite (C).

S-retest-All. When $P$ is modified, creating $P^{\prime}$, we simply reuse all runnable test cases in $T$ to test $P^{\prime}$; this is known as the retest-all technique [23]. It is often used in industry [25] and as a control technique in regression testing experiments.

S-selection. The retest all technique can be expensive: rerunning and rechecking the outcome of all test cases may require an unacceptable amount of time or human effort. Regression test selection techniques [5], [10], [24], [33] use information about $P, P^{\prime}$, and $T$ to select a subset of $T, T^{\prime}$, with which to test $P^{\prime}$. We utilize the modified entity technique [10], which selects test cases that exercise methods, in $P$, that 1 ) have been changed in producing $P^{\prime}$ or 2) use variables or structures that have been deleted or changed in producing $P^{\prime}$.

C-selection-k. Similar in concept to S-selection, this technique executes all DUTs, carved with a k-bounded reachable projection, that exercise methods that were changed in $P^{\prime}$. This technique follows the conjecture that deeper references are often not required for replay, so bounding the carving depth may improve the CR efficiency while maintaining a DUT's strengths. Within this technique, we explore depth bounding levels of 1,5 , and $\infty$ (unlimited depth which corresponds to the interface reachable projection).

C-selection-mayref. Similar to $C$-selection- $k$ except that it carves DUTs utilizing a may-reference reachable projection. This technique is based on the notion that a program change will mostly affect the parts of the heap reachable by the method under test or by the methods invoked by the method under test.

C-selection-touched. Similar to $C$-selection- $k$ except that it carves DUTs utilizing a touched-carving projection. This technique is based on the idea that modifications to the program are more likely to affect parts of the heap actually touched in the process of invoking the method under test. The touched-carving projection here is bounded to a depth of at least 1 so that the generated DUTs store at least all fields of primitive types.

\subsection{Measures}

Regression test selection techniques achieve savings by reducing the number of test cases that need to be executed on $P^{\prime}$, thereby reducing the effort required to retest $P^{\prime}$. We conjecture that $\mathrm{CR}$ techniques achieve additional savings by focusing on some methods of $P^{\prime}$. In other words, while system test case selection identifies the relevant test cases, $\mathrm{CR}$ adds another orthogonal dimension by identifying what methods are relevant.

To evaluate these effects, we measure the time to execute and the time to check the outputs of the test cases in the original test suite, the selected test suite, and the carved selected test suites. For a carved test suite, we also measure the time and space to carve the original DUT test suite. By default, we applied the default lossless filter on all DUT test suites so that DUTs with unique prestates are kept for each program method. One potential cost of regression test selection is the cost of missing faults that would have been exposed by the system tests prior to test selection. Similarly, DUTs may miss faults due to the type of change that render a DUT unexecutable or to the use of projections aimed at improving carving efficiency. We will measure fault detection effectiveness by computing the percentage of faults found by each test suite. We will also qualify our findings by analyzing instances where the outcomes of a carved test case are different from its corresponding system test case.

To evaluate the robustness of the carved test cases in the presence of program changes, we are interested in considering three potential outcomes of replaying a $D U T_{x m}$ on unit $m^{\prime}$ : 1) fault is detected, $D U T_{x m}$ causes $m^{\prime}$ to reveal a behavioral differences due to a fault; 2) false difference is detected, $D U T_{x m}$ 
TABLE 1

Siena's Component Attributes

\begin{tabular}{|l|c|c|c|c|c|}
\hline Version & SLOC & Methods & $\begin{array}{c}\text { Changed } \\
\text { methods } \\
\text { covered }\end{array}$ & $\begin{array}{c}\text { Tests } \\
\text { executing } \\
\text { changed } \\
\text { methods }\end{array}$ & Faults \\
\hline \hline v0 & 6022 & 109 & - & - & - \\
v1 & 5848 & 100 & 2 & 494 & 3 \\
v5 & 6098 & 111 & 2 & 494 & 1 \\
v6 & 6066 & 111 & 2 & 8 & 1 \\
v7 & 6034 & 107 & 10 & 503 & 2 \\
\hline
\end{tabular}

causes $m^{\prime}$ to reveal a behavioral change from $m$ to $m^{\prime}$ that is not a fault (not captured by $s t_{x}$ ); and 3 ) test is unexecutable, $D U T_{x m}$ is ill-formed with respect to $m^{\prime}$. Tests may be illformed for a variety of reasons (e.g., object protocol changes internal structure of object changes, invariants changes) and we refer to the degree to which a test set becomes ill-formed under a change as its sensitivity to change. We assess robustness by computing the percentage of carved tests and program units falling into each one of the outcomes. Since the robustness of a test case depends on the change, we qualify robustness by analyzing the relationship between the type of change and sensitivity of the DUTs.

\subsection{Artifact}

The artifact we will use to perform this experiment study is Siena [9]. Siena is an event notification middleware implemented in Java. This artifact is available for download in the Subject Infrastructure Repository (SIR) [16], [31]. SIR provides Siena's source code, a system-level test suite with 503 unique test cases, multiple versions corresponding to product releases, and a set of seeded faults in each version (the authors were not involved in this latest activity). For this study, we consider Siena's core components (not the application included in the package that is built with those components).

We utilize the five versions of Siena that have seeded faults that did not generate compilation errors (faults that generated compilation errors cannot be tested) and that were exposed by at least one system test case (faults that were not found by system tests would not affect our assessment). For brevity, we summarize the most relevant information to our study in Table 1 and point the reader to SIR [31] to obtain more details about the process employed to prepare the Siena artifact for the empirical study. Table 1 provides the number of methods, methods changed between versions and covered by the system test suite, system tests covering the changed methods, and faults included in each version. It also provides the number of physical source lines of code (SLOC) which was obtained using the $w c$ utility.

\subsection{Study Setup and Design}

The activities in this study were performed on an Opteron 250 processor, with 4 Gbytes of RAM, running Linux-Fedora, and Java 1.5. The overall process consisted of the following steps as shown in Fig. 10. First, we prepare the base test suites, System tests, $C-k^{*}, C$-mayref, and $C$-touched. The preparation of the system-level test suite was trivial because it was already

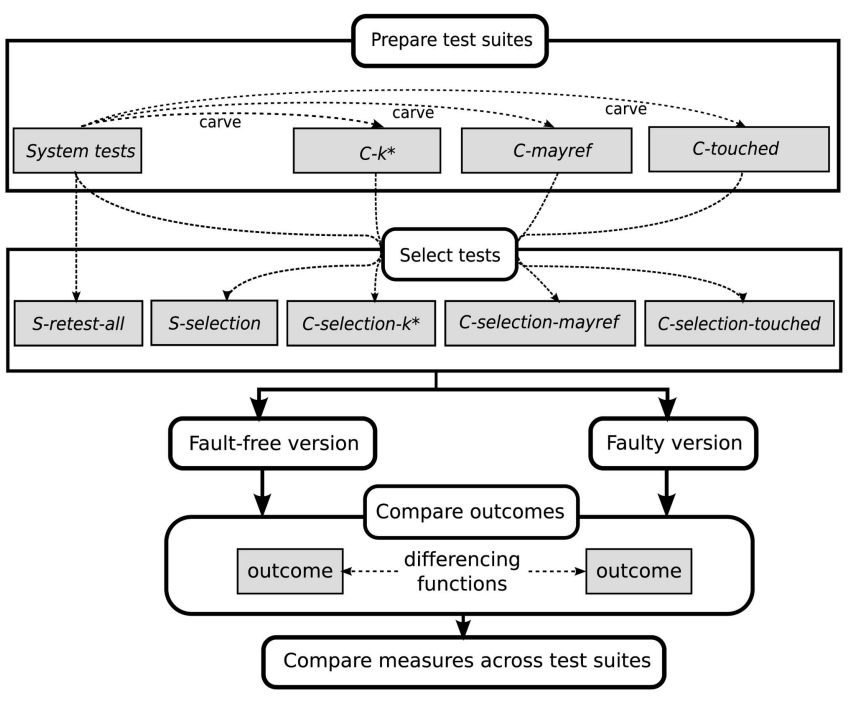

Fig. 10. Study process.

available in the repository. The preparation of the carved selection suites, required us to run the CR tool to carve all the DUTs for all the methods in $v 0$ executed by the system tests. Once the base test suites were generated, we performed test selection for each version, as described in Section 4.1, to obtain $S$-retest-all, $S$-selection, $C$-selection- $k^{*}, C$-selection-mayref, and $C$-selection-touched.

Second, we run each generated test suite on the fault-free versions of Siena to obtain an oracle for each version. For the system tests, the oracle consisted of the set of outputs generated by the program. For the carved tests, the oracle consisted of the method return value and the relevant $s_{\text {post }}$.

Third, we run each test suite on each faulty instance of each version (some versions contained multiple faults) and recorded their execution time. We dealt with each fault instance individually to control for potential masking effects among faults that might obscure the fault detection performance of the tests.

Fourth, for each test suite, we compared the outcome of each test case between the fault-free version (oracle) and the faulty instances of each version. To compare the system test outcomes between correct and faulty versions, we used predefined differencing functions that are part of our implementation which ignore transient output data (e.g., dates, times, and random numbers). For the DUTs, we performed a similar differencing, but applied to the target method return values and $s_{\text {post }}$. When the outcome of a system test case differed between the fault-free and the faulty version, a fault is said to be found. For the differences on the carved tests, we performed a deeper analysis to determine whether the observed behavioral differences correspond to faults.

Last, we compared all measures introduced in Section 4.2 across the test suites generated by $S$-retest-all, $S$-selection, $C$-selection- $k^{*}, C$-selection-mayref, and $C$-selection-touched. We then repeated the same steps to collect data for the same techniques when utilizing an of-the-shelf compression package to reduce the size of the $s_{\text {pre }}$. The results emerging from this comparison are presented in the next section. 
TABLE 2

Carving Times and Sizes to Generate Initial DUT Suites

\begin{tabular}{|l|l|c|c|c|c|c|}
\hline Carving & \multirow{2}{*}{ Metric } & \multicolumn{5}{|c|}{ Reduction } \\
\cline { 3 - 7 } & & \multicolumn{3}{|c|}{ C-select-k } & C-select & C-select \\
& & 1 & 5 & $\infty$ & mayref & touched \\
\cline { 3 - 7 } & Minutes & 120 & 120 & 120 & 122 & 120 \\
& MB & 845 & $1.8 \mathrm{~K}$ & $1.8 \mathrm{~K}$ & $1.3 \mathrm{~K}$ & $1.0 \mathrm{~K}$ \\
\hline \hline Plain & Minutes & 125 & 128 & 125 & 124 & 124 \\
& MB & 2.6 & 3.5 & 3.4 & 3.0 & 2.8 \\
\hline Numpressed & 20698 & 23399 & 23514 & 22391 & 20961 \\
\hline $\begin{array}{l}\text { Percentage of DUTs } \\
\text { with sentinels }\end{array}$ & $69 \%$ & $3 \%$ & - & $47 \%$ & $61 \%$ \\
\hline
\end{tabular}

\subsection{Results}

In this section, we provide the results for each research question regarding carving and replaying efficiency, fault detection effectiveness, and robustness and sensitivity of the DUT suites.

RQ1: Efficiency. We first focus on the efficiency of the carving process. Although our infrastructure completely automates carving, this process does consume time and storage so it is important to assess its efficiency as it might impact its adoption and scalability. Table 2 summarizes the time (in minutes) and the size (in megabytes-MB) that it took to carve and store the complete initial suite carved from $v 0$ of approximately 20,000 DUTs utilizing the different CR techniques with and without the use of compression on the $s_{\text {pre }}$ and $s_{\text {post }}$.

In the first row of Table 2, we observe that, for Siena, constraining the carving depth barely affects the carving time. However, we see that constraining the carving depth can greatly reduce the required space, as carving at $k=1$ requires 47 percent of the space required for carving with infinite depth. Observe that for depths greater than 1, the differences in storage space are small due to the rather "shallow" nature of the artifact (dereference chains with length greater than 2 are rare in Siena). C-select-mayref carving required additional time because of the extra static analysis performed up front, but consumed 55 percent of the space. Utilizing the touched-carving projection resulted in space requirements averaging those of $k=1$ and $k=\infty$. Compressing the stored DUTs with the open source utility bzip provided space savings of 99.7 percent when carving at unlimited depth, but added 4-8 minutes over the whole test suite carving process.

The last two rows of Table 2 reveal that 69 percent of the DUTs carved at $k=1$ contained sentinels while only 3 percent of the DUTs carved at a $k=5$ contained sentinels. The differences in sentinels mean that deeper differences in the heap are more often obscured by using $k=1$, which explains why filtering is more effective on the smaller space captured by $k=1$. The touched suite size and carving costs resemble those of $k=1$, while the mayref size and costs fit in between those of $k=1$ and $k=5$.

It is important to note that the carving numbers reported in Table 2 correspond to the initial carving of the complete DUT suite-DUTs carved for each of the methods in Siena from each of 503 system tests that may execute each method. Carving was performed automatically without the

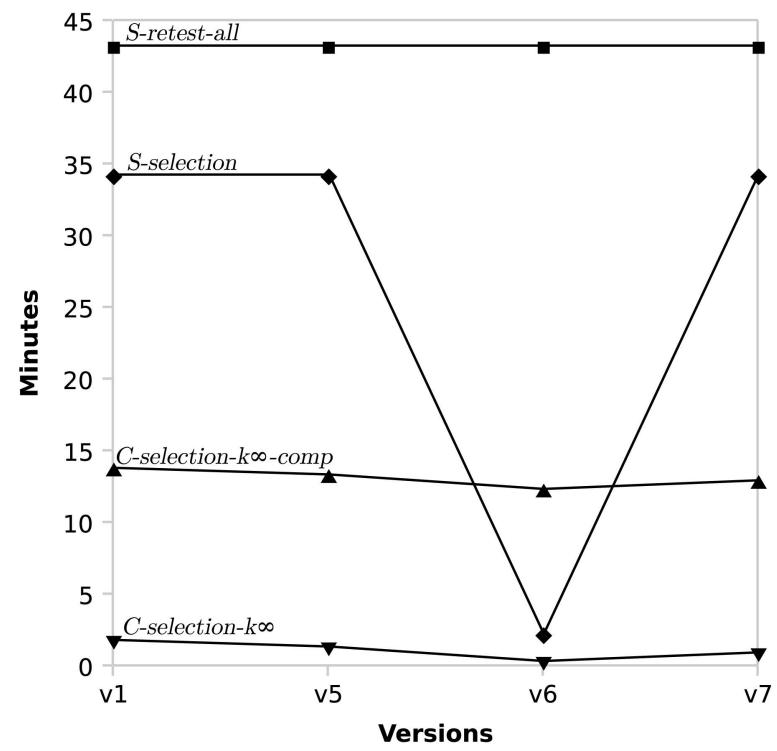

Fig. 11. Test suite execution times for system test suites and $C$-selection- $k \infty$ (with and without compression).

tester's participation. As with regular unit tests, during the evolution of the system, DUTs will be replayed repeatedly amortizing the initial carving costs, and only a subset of the DUTs will need to be recarved (e.g., recarving the DUTs affected by the changes in $v 6$ would only require 2 percent of the original carving time). Recarving will be necessary when it is determined that changes in the program may affect a DUT's relevant prestate.

We now proceed to analyze replay efficiency. Replay efficiency is particularly important since, as with regular units tests, it is likely that a carved DUT will be repeatedly replayed as a method evolves while preserving its original intended behavior.

Fig. 11 shows the time in minutes to execute the system regression test suites and to replay the $C$-selection- $k \infty$ suite (the most expensive of all carved suites). Each observation corresponds to the replay time of each generated test suite under each version, while the lines joining observations are just meant to assist in the interpretation.

Replaying the $C$-selection- $k \infty$ provides gains of at least an order of magnitude over the $S$-select suites, averaging less than a minute per version. On average, replaying carved suites take 2 percent of the time required by $S$-retest-all, and 3 percent of the time required by $S$-select. Utilizing $C$ selection- $k \infty$-comp incurs a large overhead to uncompress the DUTs content, rendering its application unlikely in spite of the storage savings. The test suite resulting from the $S$-retest-all technique consistently averages 43 minutes per version. The test suites resulting from $S$-select averages 28 minutes across versions, with savings over $S$-retest-all ranging from barely a minute in $v 7$ to a maximum of 41 minutes in $v 6 .^{1}$

We also measured the diffing time required by all techniques. For the system test suites the diffing times were consistently less than a minute, and for the

1. Factors that affect the efficiency of this technique are not within the scope of this paper but can be found in [18]. 


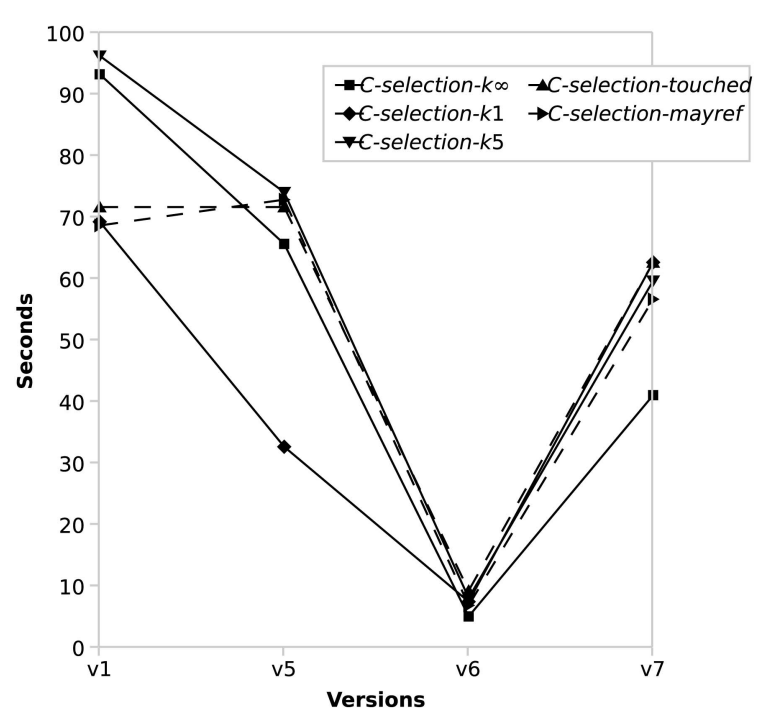

Fig. 12. Test suite execution times for the $C$-selection- $k^{*}$ suites.

$C$-selection* suites the time never exceeded 15 seconds, making both negligible compared with the replay time.

Fig. 12 summarizes the replay execution times for some of the other test suites we generated. We find that, on average, all the $C$-selection* suites (excluding the one with compression) replay execution time was less than 1 minute. They all took less than 10 seconds to replay $v 6$ and up to 96 seconds to replay the DUTs selected for $v 1$. Constraining the carving depth with $k=1$ consistently reduced replay time (over percent 50 reduction in v5). Similarly, constraining the carving space through either $C$-selection-mayref or $C$-selection-touched reduced the replay time in some versions (almost 20 percent reduction in $v 1$ ).

RQ2: Fault detection effectiveness. Most of the test suites carved from $S$-selection, (with $k \geq 1$ ), C-selectionmayref, and $C$-selection-touched detected as many faults as the $S$-retest-all technique. This indicates that a DUT test suite can be as effective as a system test suite at detecting faults, even when using aggressive projections.

It is worth noting, however, that computing fault detection effectiveness over a whole DUT suite overlooks the fact that, for some system tests, their corresponding carved DUTs may have lost or gained fault detection effectiveness. We conjecture that this is a likely situation with our artifact because many of the faults are detected by multiple system tests, so there were many carved DUTs that could have detected each fault. To address this situation, we perform an effectiveness analysis at the test case level. For each carving technique we compute: 1) PP, the percentage of passing selected system tests (selected utilizing S-Selection) that have all corresponding DUTs passing, and 2) FF, the percentage of failing system tests that have at least one corresponding failing DUT. Table 3 presents the PP and FF values for the suites under all faults in each version. In general, we observe that most PP and FF values are over 90 percent indicating that DUTs carved from a system test case tend to preserve much of their effectiveness. But, we can also identify some interesting exceptions. For example, independent of the DUT suite, for $v 7: f 1$ (the first fault in version $v 7$ ), only 24 percent of the passing system tests had all their associated DUTs passing. The rest of the system tests had at least one DUT that detected a behavioral difference that was not detected by the system test case oracle because it did not propagate to the output (the level at which the system test case oracle operated). This is one example where a DUT is more powerful than its corresponding system test.

Another interesting instance is FF for $C$-selection- $k 1, v 5$, where we observed that replaying the carved test suite did not detect any of the behavioral differences exhibited by the selected system test cases. Upon further examination, we found that the changed method in $v 5$ required access to references in the heap deeper than $k=1$ which were not satisfied by the captured prestate of the $C$-selection- $k 1$ suite, therefore resulting in a SentinelAccessException. Because of this, no poststates were stored for the method and the fault goes undetected. The other carved test suites on $v 5$ did detect the fault since they either carved deeper prestates or, in the case of the touched-carving projection test suite, carved the parts of the prestates that were necessary for the methods under test. Still, for the other suites on $v 5,3$ out of the 300 failing system tests did not have any corresponding DUT on the changed methods failing (99 percent). We observed a similar situation in $v 7$ : $f 2$ where 18 out of 203 DUTs (9 percent) did not expose behavioral differences even though the corresponding system tests failed. When we analyzed the reasons for this reduction in $\mathrm{FF}$, we discovered that in both cases the tool did not carve in $v 0$ the prestate for one of the changed methods because the system test case did not reach them;

TABLE 3

Fault Detection Effectiveness

\begin{tabular}{|c|c|c|c|c|c|c|c|c|c|c|}
\hline & \multicolumn{5}{|c|}{$\mathrm{PP}$} & \multicolumn{5}{|c|}{$\mathrm{FF}$} \\
\hline & \multicolumn{3}{|c|}{ C-selection-k } & \multirow{2}{*}{$\begin{array}{c}\text { C-selection } \\
\text { mayref }\end{array}$} & \multirow{2}{*}{$\begin{array}{c}\text { C-selection } \\
\text { touched }\end{array}$} & \multicolumn{3}{|c|}{ C-selection-k } & \multirow{2}{*}{$\begin{array}{c}\text { C-selection } \\
\text { mayref }\end{array}$} & \multirow{2}{*}{$\begin{array}{c}\text { C-selection } \\
\text { touched }\end{array}$} \\
\hline & 1 & 5 & $\infty$ & & & 1 & 5 & $\infty$ & & \\
\hline "v1:f1 & $\overline{100}$ & $\overline{100}$ & 100 & 100 & $\overline{100}$ & $\overline{100}$ & $\overline{100}$ & $\overline{100}$ & $\overline{100}$ & 100 \\
\hline $\mathrm{v} 1: \mathrm{f} 2$ & 100 & 100 & 100 & 100 & 100 & 100 & 100 & 100 & 100 & 100 \\
\hline $\mathrm{v} 1: \mathrm{f} 3$ & 100 & 100 & 100 & 100 & 100 & 100 & 100 & 100 & 100 & 100 \\
\hline v5 & 100 & 100 & 100 & 100 & 100 & 0 & 99 & 99 & 99 & 99 \\
\hline v6 & 100 & 100 & 100 & 100 & 100 & 100 & 100 & 100 & 100 & 100 \\
\hline v7:f1 & 24 & 24 & 24 & 24 & 24 & 100 & 100 & 100 & 100 & 100 \\
\hline v7:f2 & 100 & 100 & 100 & 100 & 100 & 91 & 91 & 91 & 91 & 91 \\
\hline Average & 89 & 89 & 89 & 89 & 89 & 84 & 99 & 99 & 99 & 99 \\
\hline
\end{tabular}


TABLE 4

Robustness and Sensitivity

\begin{tabular}{|l|c|c|}
\hline & \multicolumn{2}{|c|}{ \% of DUTs Detecting a Difference in } \\
\cline { 2 - 3 } & Changed Methods & Faulty Changed Methods \\
\hline \hline v1:f1 & 3 & 20 \\
v1:f2 & 20 & 3 \\
v1:f3 & 15 & 100 \\
v5 & 7 & 7 \\
v6 & 0 & 100 \\
v7:f1 & 0 & 100 \\
v7:f2 & 0 & 100 \\
\hline
\end{tabular}

call graphs generated for the system test cases indicate that the faulty methods were not invoked during the execution of some of the system test cases on $v 0$ of Siena. Changes in the code structure (e.g., addition of a method call), however, made the system test cases reach those changed methods (and expose a fault) in later versions. In both circumstances, improved DUTs that would have resulted in 100 percent FF could have been generated by recarving the test cases in later versions (carve from $v_{i}$ instead of $v 0$ to replay in $v_{i+1}$ ). More generally, these observations point out again for the need for mechanisms to detect changes in the code that should trigger recarving.

RQ3: Robustness and sensitivity. We examined how DUTs obtained through $C$-selection- $k 1$ are quite fragile in terms of their executability, and how certain code changes may make a method reach a new part of the heap that was not originally carved. We further evaluate the robustness and sensitivity of DUTs by comparing their performance in the presence of methods that changed but are not faulty and in the presence of methods that changed and are indeed faulty. We performed such detailed comparison on the suites generated with $C$-selection- $k \infty$. Table 4 summarizes the findings and we now briefly discuss distinct instances of the scenarios we found.

In both faulty instances of $v 7$, the version with the most methods changed (10), none of the DUTs revealing behavioral differences were found by methods other than the faulty ones. This is clearly an ideal situation, which is also present in $v 6$. $V 1: f 3$ represents perhaps a more common case where 15 percent of the DUTs going through nonfaulty changed methods detected differences, but 100 percent of the DUTs traversing faulty methods actually revealed a poststate difference. $V 1: f 2$ presents a scenario in which carving generates more behavioral differences for the nonfaulty changed methods than for the faulty changed methods, showing that even for correct changes the number of affected DUTs may be large (13 out of 65 ). In this case, the implementation change was such that the method switched the order of division and multiplication operations involving a variable which was eventually returned. Because of this, there was a difference in the return value, which was detected as a behavioral difference, and would probably be detected by other forms of unit tests as well.

It is worth noting that the differencing functions offer an opportunity to control this problem. For example, a more relaxed differencing mechanism focused on just return values could have detected the fault while reducing the number of false differences if the fault manifests itself in the return value. Mechanisms to select and appropriately combine these differencing functions will be important for the robustness and sensitivity of DUTs. In addition, we anticipate that as the CR components of the framework become parts of an IDE, the additional change information available in the developer's environment could help to reduce the number of false positives. For example, code modifications due to refactoring that do not affect the target unit's interface would be expected to retain the same behavior. However, changes that can be mapped to the bug repository would be expected to affect the unit's behavior.

\subsection{Targeted Case Studies}

The previous study addressed the stated research questions with respect to Siena, and we believe the findings generalize to similar artifacts. Still, we realize that our study suffers from threats to validity. Specifically, the selected artifact provided limited exposure to CR in the presence of deeper heap structures, extensive software changes, and high number of methods invocations. We have started to address those threats to the validity of our findings by investigating the performance of $C R$ in the presence of such settings. More specifically, we have studied the performance of CR on two other artifacts, NanoXML and Jtopas [16], that provide exposure to more complex heap structures, highfrequency executions sequences, and extensive changes between versions. These studies confirm our previous findings, but also show that the performance of the different carving strategies can vary significantly in programs with complex heap structures, that the Replay AnomalyHandler can enhance DUTs reuse and potential for fault detection with affordable replay costs, and that the clustering projection can be very effective to reduce the number of DUTs on high-frequency methods. Due to space constraints, the detailed settings and results are omitted here but available in a technical report [12].

\section{Related Work}

Our work was inspired by Weide's notion of modular testing as a means to evaluate the modular reasoning property of a piece of software [37]. Although Weide's focus was on the evaluation of the fragility of modular reasoning, he raised some important questions regarding the potential applicability of what he called a "modular regression technique" that led to our work.

Within the context of regression testing, our approach is similar to Binkley's semantic guided regression testing in that it aims to reduce testing costs by running a subset of the program [5], [6]. Binkley's technique utilizes static slicing to identify potential semantic differences between two versions of a program. He also presents an algorithm to identify the system tests that must be run on the slices resulting from the differences between the program versions. The fundamental distinction between this and our approach is that we do not run system-level tests, but rather smaller and more focused unit tests. Another important distinction is that our targets are not the semantic differences between versions, but rather methods in the program. 
The preliminary results from our original test carving prototype [29] evidenced the potential of carved tests to improve the efficiency and the focus of a large system test suite, identified challenges to scale up the approach, and pinpoint scenarios under which the carved test cases would and would not perform well. We have built on that work by presenting a generic framework for differential carving, extending the type of analysis we performed to make the approach more scalable, and by developing a full set of tools that can enable us to explore different techniques on various programs.

We are aware of other research efforts related to the notion of test carving. First, Orso and Kennedy's and Clause et al.'s notion of selective capture and replay of program executions [26]. Orso and Kennedy's technique [26] aims to selectively capture and replay events and interactions between the selected program components and the rest of the application. The technique captures a simplified state representation composed of the object IDs, types, and scalar values directly utilized by the selected program components to enable replay. The approach is similar to carving with a touched projection with the difference that simplified heap structures are used to represent the program state.

Second, the test factoring approach introduced by Saff and Ernst takes a similar approach to Orso's with the creation of mock objects that serve to create the scaffolding to support the execution of the test unit [35]. The same group introduced a tool set for a fully featured Java execution environments that can handle many of the subtle interactions present in this programming language (e.g., callbacks, arrays, and native methods) [34]. Saff et al.'s work [34] carves a method test case by recording the sequence of calls that can influence the method, the sequence of calls made by the method, and the return values and unit state side effects of those calls. In our framework, this would amount to calculating $\bar{\sigma}$ such that $s(\bar{\sigma})=s_{\text {pre }}$ for the method of interest and then calculating summarizing traces $\bar{\sigma}_{\text {call }_{i}}$ that reflect the return value and side effects for each call out of the method and carving $s_{p r e}$, the relevant prestate for each call. During replay, the same sequence of calls with the same parameters is expected-any deviation results in a report of a difference during replay. In our framework, we would identify the points at which the $n$ calls out of the method occur as poststate locations to define a DUT of the form $\left(\bar{\sigma},\left(s_{\text {pre }_{1}}, \ldots, s_{\text {pre }_{n}}\right)\right)$.

The approaches introduced by Orso et al. and Saff et al. are action-based approaches that capture the interactions between the target unit and its context and then build the scaffolding to replay just those interactions. Hence, they do not incur in costs associated with capturing and storing the system state for each targeted unit. On the other hand, these approaches are likely to generate inefficient unit tests in the presence of long-running system tests and they may generate tests that are too sensitive to simple changes that do not effect meaning of the unit (e.g., changing the order of independent method calls). Saff et al. have identified this issue and propose to analyze the life span of the factored test cases across sequences of method modifications [34]. This is a critical factor in judging the cost-effectiveness of CR testing, and we have started to study it in Section 4.5. In terms of our framework, both of these approaches would be considered action-based CR approaches. We have presented, what is to the best of our knowledge, the first statebased approach to CR testing.

More recently, $\mathrm{Xu}$ et al. have proposed a hybrid approach that mixes action based with state based to enhance replay efficiency [41]. The approach only captures the set of runtime values required to reach a checkpoint and the values that could potentially be required to complete execution after the checkpoint. The set of runtime values required is obtained by computing the slice of the program required to generate those values (similar to action based). The set of values that could be required to complete execution is computed by walking the heap (similar to state based). In our framework, such a test could be defined by calculating traces $\bar{\sigma}_{\text {control }_{i}}$ leading to checkpoint $s_{\text {pre }}$ and a number of states $s_{\text {post }}$ corresponding to the method return points. This would result in a DUT of the form $\left(\left(\bar{\sigma}_{1}, \ldots, \bar{\sigma}_{j}\right), s_{\text {pre }},\left(s_{\text {post }_{1}}, \ldots, s_{\text {post }_{h}}\right)\right)$, where $j$ is the number of relevant subtraces that lead to the checkpoint stack and $h$ is the number of states that affect the postcheckpoint program execution.

All of these related efforts have shown their feasibility in terms of being able to replay tests and Saff et al.'s and Xu et al.'s approaches have provided initial evidence that they can save time and resources under several scenarios. None of these approaches, however, has been evaluated in terms of its fault detection effectiveness which ultimately determines the value of the carved tests, or in the context of regression testing.

Our work also relates to efforts aimed at developing unit test cases. Several frameworks grouped under the umbrella of Xunit have been developed to support software engineers in the development of unit tests. JUnit, for example, is a popular framework for the Java programming language that lets programmers attach testing code to their classes to validate their behavior [19].

There are also multiple approaches that automate, to different degrees, the generation of unit tests. For example, commercial tools such as Jtest, developed by a company called Parasoft, develop unit test cases by analyzing method signatures and selecting test cases that increase some coverage criteria [22]. Some of these tools aim to assess software robustness (e.g., whether an exception is thrown [13]). Others utilize some type of specification such as pre and postconditions or operational abstractions, to guide the test case generation and actually check whether the test outcome meets the expectation results [7], [11], [27], [39]. Interestingly enough, a part of JTest called JTest Tracer can be used to monitor a deployed application in real time and capture inputs to generate realistic JUnit test cases [22], a process somewhat similar to carving.

Although carving also aims to generate unit test cases, the approach we propose is different from previous unit test case generation mechanisms since it consists of the projection of a system test case onto the targeted software unit. As such, we expect for carved unit tests to retain some of the interesting interactions exposed by systems tests. In general, such interactions are hard to design and are rarely included in regular unit test cases. 
As stated, the poststate differencing functions that regulate the detection of differences between encodings of unit behavior belongs to a larger body of testing work on differential-based oracles. For example, the work of Weyuker [38] on the development of pseudo-oracles, Jaramillo et al. [21] on using comparisons to check for optimization induced errors in compilers, or the comparison of program spectra [30] are instances of utilizing differencing-type oracles at the system or subsystem level. When focusing at the unit level of object-oriented programs, as we are doing, Binder suggests the term "concrete state" oracles, which aim to compare the value of all the unit's attributes against what is expected [4]. Briand et al. referred to this type of oracle as a "precise" oracle because it was the most accurate one employed in their studies [8]. Overall, the notion of testing being fundamentally differential has long been understood [38], since the pseudo-oracles against which systems are judged correct are themselves subject to error. Thus, the question we aimed to answer is not whether our CR method judges a system correct or incorrect, but rather whether it is capable of cost-effectively detecting differences between encodings of system behavior that developers can easily mine to judge whether the difference reflects an error.

\section{Conclusion}

We have presented a general framework for automatically carving and replaying DUTs. The framework accommodates two types of state representation, and incorporates sophisticated projection, anomaly handling, and differencing strategies that can be instantiated in various ways to suit distinct trade-offs. We have implemented a state-based instance of the framework that mitigates testing costs through a family of reachability-based projections, that enhances DUT robustness through replay anomaly handlers, and that can adjust the sensitivity of DUTs through differencing functions.

Our evaluation of this implementation on Siena, NanoXML, and JTopas provides evidence that DUTs can be generated automatically from system tests, can provide efficiency gains of orders of magnitude while retaining most of the effectiveness of system tests in a regression testing context, and can be robust to program changes and scale to large and complex heap structures.

The experiences gained while instantiating and assessing the framework suggest several directions for future work. First, we will perform further studies not only to confirm our findings on other artifacts under similar settings but also to compare DUTs with traditional unit tests developed by software engineers. We conjecture that software engineers develop rather shallow unit tests and that we can effectively complement those with DUTs that expose the target units to more complex execution settings.

A longer-term direction is the exploration of other transformation techniques that utilize our current test representation. For example, we are investigating automated mechanisms that combine multiple DUTs to create an aggregated DUT for a larger program unit such as a class. This could be achieved by clustering multiple DUTs based on the identity of the receiver object, effectively transferring the effects of methods on the receiver object throughout the sequence, achieving a kind of interaction testing between calls. Ultimately, we envision a family of automated transformations of testing resources where carving is just one of those transformations.

\section{ACKNOWLEDGMENTS}

This work was supported in part by the US National Science Foundation through CAREER award 0347518, CCF awards 0429149 and 0541263, CRI award 0454203, an IBM Eclipse Innovation Award, the Lockheed Martin Software Technology Initiative, and by the US Army Resarch Office through DURIP award W911NF-04-1-0104. The authors would like to thank B. Weide for inspiring this effort, S. Reddy for the feasibility exploration she provided through her thesis, and J. Dokulil for his effort in the initial implementation of the CR tool. They would also like to thank V. Ranganath for supporting their use of Indus and O. Tkachuk for implementing preliminary versions of the static analysis.

\section{References}

[1] J. Bach, "Useful Features of a Test Automation System (Part III)," Testing Techniques Newsletter, Oct. 1996.

[2] K. Beck, Extreme Programming Explained: Embrace Change, first ed. Addison-Wesley Professional, Oct. 1999.

[3] K. Beck, Test Driven Development: By Example. Addison Wesley Longman, Nov. 2002.

[4] R. Binder, Testing Object-Oriented Systems: Models, Patterns, and Tools, chapter 18, Object Technologies, pp. 943-951, first ed. Addison Wesley, Oct. 1999.

[5] D. Binkley, "Semantics Guided Regression Test Cost Reduction," IEEE Trans. Software Eng., vol. 23, no. 8, pp. 498-516, Aug. 1997.

[6] D. Binkley, R. Capellini, L. Ross Raszewski, and C. Smith, "An Implementation of and Experiment with Semantic Differencing," Proc. IEEE Int'l Conf. Software Maintenance, pp. 82-91, Nov. 2001.

[7] C. Boyapati, S. Khurshid, and D. Marinov, "Korat: Automated Testing Based on Java Predicates," Proc. Int'l Symp. Software Testing and Analysis, pp. 123-133, July 2002.

[8] L.C. Briand, M. Di Penta, and Y. Labiche, "Assessing and Improving State-Based Class Testing: A Series of Experiments," IEEE Trans. Software Eng., vol. 30, no. 11, pp. 770-793, Nov. 2004.

[9] A. Carzaniga, D. Rosenblum, and A. Wolf, "Achieving Scalability and Expressiveness in an Internet-Scale Event Notification Service," Proc. 19th Ann. ACM Symp. Principles of Distributed Computing, pp. 219-227, July 2000.

[10] Y.-F. Chen, D.S. Rosenblum, and K.-P. Vo, "TestTube: A System for Selective Regression Testing," Proc. 16th Int'l Conf. Software Eng., pp. 211-220, May 1994.

[11] Y. Cheon and G.T. Leavens, "A Simple and Practical Approach to Unit Testing: The JML and JUnit," Proc. 16th European Conf. ObjectOriented Programming, pp. 231-255, June 2002.

[12] H.N. Chin, S. Elbaum, M.B. Dwyer, and M. Jorde, "DUTs: Targeted Case Studies," Technical Report TR-UNL-CSE-20070005, Univ. of Nebraska, Aug. 2008.

[13] C. Csallner and Y. Smaragdakis, "Jcrasher: An Automatic Robustness Tester for Java," Software Practice and Experience, vol. 34, no. 11, pp. 1025-1050, Sept. 2004.

[14] M. Dahm and J. van Zyl, Byte Code Engineering Library, http:// jakarta.apache.org/bcel/, June 2002.

[15] S. Dieckmann and U. Holzle, "A Study of the Allocation Behavior of the Specjvm98 Java Benchmark," Proc. 13th European Conf. Object-Oriented Programming, pp. 92-115, June 1999.

[16] H. Do, S.G. Elbaum, and G. Rothermel, "Supporting Controlled Experimentation with Testing Techniques: An Infrastructure and Its Potential Impact," Empirical Software Eng.: An Int'l J., vol. 10, no. 4, pp. 405-435, Oct. 2005.

[17] S. Elbaum, H. Nee Chin, M.B. Dwyer, and J. Dokulil, "Carving Differential Unit Test Cases from System Test Cases," Proc. ACM SIGSOFT Symp. Foundations of Software Eng., pp. 253-264, Nov. 2006 . 
[18] S. Elbaum, P. Kallakuri, A.G. Malishevsky, G. Rothermel, and S. Kanduri, "Understanding the Effects of Changes on the CostEffectiveness of Regression Testing Techniques," J. Software Testing, Verification, and Reliability, vol. 13, no. 2, pp. 65-83, June 2003.

[19] E. Gamma and K. Beck, JUnit, http://sourceforge.net/projects/ junit, Dec. 2005.

[20] R. Hildebrandt and A. Zeller, "Simplifying Failure-Inducing Input," Proc. Int'l Symp. Software Testing and Analysis, pp. 135145, Aug. 2000.

[21] C. Jaramillo, R. Gupta, and M.L. Soffa, "Comparison Checking: An Approach to Avoid Debugging of Optimized Code," Proc. European Software Eng. Conf./Foundations of Software Eng., pp. 268284, Sept. 1999.

[22] JTest, Jtest Product Overview, http://www.parasoft.com/jsp/ products/home.jsp?product=Jtest, Oct. 2005.

[23] H.K.N. Leung and L. White, "Insights into Regression Testing," Proc. IEEE Int'l Conf. Software Maintenance, pp. 60-69, Oct. 1989.

[24] H.K.N. Leung and L. White, "A Study of Integration Testing and Software Regression at the Integration Level," Proc. IEEE Int'l Conf. Software Maintenance, pp. 290-300, Nov. 1990.

[25] A.K. Onoma, W.-T. Tsai, M. Poonawala, and H. Suganuma, "Regression Testing in an Industrial Environment," Comm. ACM, vol. 41, no. 5, pp. 81-86, May 1998.

[26] A. Orso and B. Kennedy, "Selective Capture and Replay of Program Executions," Proc. Third Int'l Workshop Dynamic Analysis, May 2005.

[27] C. Pacheco and M.D. Ernst, "Eclat: Automatic Generation and Classification of Test Inputs," Proc. 19th European Conf. ObjectOriented Programming, pp. 504-527, July 2005.

[28] V.P. Ranganath and J. Hatcliff, "Pruning Interference and Ready Dependence for Slicing Concurrent Java Programs," Proc. 13th Int'l Conf. Compiler Construction, pp. 39-56, Apr. 2004.

[29] S.K. Reddy, "Carving Module Test Cases from System Test Cases: An Application to Regression Testing," master's thesis, Dept. of Computer Science and Eng., Univ. of Nebraska, July 2004.

[30] T. Reps, T. Ball, M. Das, and J. Larus, "The Use of Program Profiling for Software Maintenance with Applications to the Year 2000 Problem," Proc. European Software Eng. Conf./Foundations of Software Eng.), pp. 432-449, Sept. 1997.

[31] G. Rothermel, S. Elbaum, and H. Do, Software Infrastructure Repository, http://cse.unl.edu/galileo/php/sir/index.php, Jan. 2006.

[32] G. Rothermel, S. Elbaum, A.G. Malishevsky, P. Kallakuri, and X. Qiu, "On Test Suite Composition and Cost-Effective Regression Testing," ACM Trans. Software Eng. and Methodologies, vol. 13, no. 3, pp. 277-331, July 2004.

[33] G. Rothermel and M.J. Harrold, "Analyzing Regression Test Selection Techniques," IEEE Trans. Software Eng., vol. 22, no. 8, pp. 529-551, Aug. 1996.

[34] D. Saff, S. Artzi, J. Perkins, and M. Ernst, "Automated Test Factoring for Java," Proc. 20th Ann. Int'l Conf. Automated Software Eng., pp. 114-123, Nov. 2005.

[35] D. Saff and M. Ernst, "Automatic Mock Object Creation for Test Factoring," Proc. SIGPLAN/SIGSOFT Workshop Program Analysis for Software Tools and Eng., pp. 49-51, June 2004.

[36] D. Saff and M.D. Ernst, "An Experimental Evaluation of Continuous Testing During Development," Proc. Int'l Symp. Software Testing and Analysis, pp. 76-85, July 2004.

[37] B. Weide, "Modular Regression Testing: Connections to Component-Based Software," Proc. Fourth ICSE Workshop ComponentBased Software Engineering, pp. 82-91, May 2001.

[38] E.J. Weyuker, "On Testing Non-Testable Programs," The Computer J., vol. 25, no. 4, pp. 465-470, Nov. 1982.

[39] T. Xie and D. Notkin, "Tool-Assisted Unit-Test Generation and Selection Based on Operational Abstractions," Automated Software Eng. J., July 2006.

[40] Xstream-1.1.2, XStream, http://xstream.codehaus.org, Aug. 2005.

[41] G. Xu, A. Rountev, Y. Tang, and F. Qin, "Efficient Checkpointing of Java Software Using Context-Sensitive Capture and Replay," Proc. ACM SIGSOFT Symp. Foundations of Software Eng., pp. 85-94, Oct. 2007

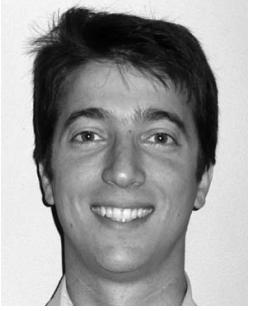

Sebastian Elbaum received the PhD degree in computer science from the University of Idaho and the degree in systems engineering from the Universidad Catolica de Cordoba, Argentina. He is an associate professor at the University of Nebraska, Lincoln. His research interests include program analysis and testing, deployed software analysis, user software engineering, and empirical software engineering. He received the US National Science Foundation Faculty Early CAREER Award in 2004 for his research on the utilization of field data to test highly configurable and rapidly evolving pervasive systems. $\mathrm{He}$ was the program chair for the ACM International Symposium of Software Testing and Analysis (2007) and a program cochair for the IEEE Symposium of Empirical Software Engineering and Measurement (2008). He has served as a program committee member for several international conferences, including the International Conference on Software Engineering and the Symposium on Foundations of Software Engineering. $\mathrm{He}$ is a member of the IEEE.

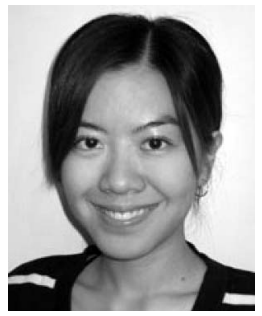

Hui Nee Chin received the BS degree and the MS degree in computer science from the University of Nebraska at Lincoln (UNL) in 2005 and 2007, respectively. She is a software engineer in the Macintosh Business Unit at Microsoft Corp. She is an alumna of the Laboratory for Empirically-based Software Quality Research and Development (ESQuaReD) at UNL. Her research interests include software testing and analysis, particularly automatic test case generation.

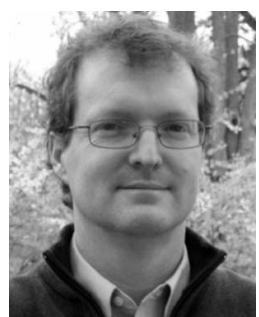

Matthew B. Dwyer received the BS degree in electrical engineering from the University of Rochester in 1985, the MS degree in computer science from the University of Massachusetts at Boston, and the PhD degree from the University of Massachusetts at Amherst. He is the Henson Professor of Software Engineering in the Department of Computer Science and Engineering at the University of Nebraska, Lincoln. He worked for six years as a senior engineer with Intermetrics Inc., developing compilers and software for safety-critical embedded systems. His research interests include software analysis, verification, and testing. He has served as a program chair for the SPIN Workshop on Model Checking of Software (2001), the ACM Workshop on Program Analysis for Software Tools and Engineering (2002), the ACM Symposium on Foundations of Software Engineering (2004), the ETAPS conference on Fundamental Approaches to Software Engineering (2007), and the International Conference on Software Engineering (2008). He is an ACM distinguished scientist. He is a member of the IEEE and the IEEE Computer Society.

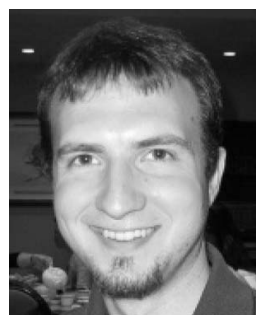

Matthew Jorde received the BS degree and the MS degree in computer science from the University of Nebraska at Lincoln (UNL) in 2006 and 2008, respectively. He is a technical analyst at Applied Underwriters, Foster City, California. $\mathrm{He}$ is an alumnus of the Jeffrey $\mathrm{S}$. Raikes School of Computer Science and Management and of the Laboratory for Empiricallybased Software Quality Research and Development (ESQuaReD) at UNL. His research interests include software testing and analysis.

$\triangleright$ For more information on this or any other computing topic, please visit our Digital Library at www.computer.org/publications/dlib. 